RUNNING HEAD: MULTISENSORY ENCODING OF NAMES

\title{
Exploiting multisensory encoding of names via name tags facilitates remembering
}

Carolyn A. Murray ${ }^{1, *}$, Maisy Tarlow ${ }^{1}$, Jesse Rissman ${ }^{1,2}$, and Ladan Shams ${ }^{1,3}$

${ }^{1}$ Dept. of Psychology

${ }^{2}$ Dept. of Psychiatry \& Behavioral Sciences

${ }^{3}$ Dept. of Bioengineering and Neuroscience Interdepartmental Program

University of California, Los Angeles

1285 Psychology Building, Los Angeles, CA, 90095

*=corresponding author; camurray@ucla.edu 


\section{MULTISENSORY ENCODING OF NAMES}

\section{Abstract}

Associating names to faces can be challenging, but it is an important task that we engage in throughout our lives. An interesting feature of this task is the lack of an inherent, semantic relationship between a face and name. Previous scientific research, as well as common lay theories, offer strategies that can aid in this task (e.g., mnemonics, semantic associations). However, these strategies are either impractical (e.g., spaced repetition) or cumbersome (e.g., mnemonics). The current study seeks to understand whether bolstering names with crossmodal cues — specifically, name tags — may aid memory for face and name pairings. In a series of five experiments, we investigated whether the presentation of congruent auditory (vocal) and written names at encoding might benefit subsequent cued recall and recognition memory tasks. The first experiment consisted of short video clips of individuals verbally introducing themselves (auditory cue), presented with or without a name tag (visual cue). The results showed that participants, cued with a picture of a face, were more likely to recall the associated name when those names were encoded with a name tag (i.e. a congruent visual cue) compared to when no supporting cross-modal cue was available. Subsequent experiments probed the underlying mechanism for this facilitation of memory. The findings were consistent with a benefit of multisensory encoding, above and beyond any effect from the availability of multiple independent unisensory traces. Overall, these results extend previous findings of a benefit of multisensory encoding in learning and memory, to a naturalistic associative memory task.

Keywords: multisensory processing, associative memory, name learning, multisensory benefit 


\section{Introduction}

As any individual who has been to a large gathering can attest, remembering the association between names and faces is a challenge. While associative memory tasks tend to be among the most challenging in a laboratory setting, memory for names is one that is considered especially so, in part due to the lack of an inherent, semantic relationship between a face and name (e.g., there isn't anything about one face that makes it seem more "Hannah" than another). This makes learning names a challenge, which has been the topic of much past research (for examples, see McWeeny et al, 1987; Cohen \& Faulkner, 1986; Brooks III et al, 1993).

Previous studies have tested a number of different approaches to improve recall of names associated with particular faces. Spacing the learning of the names has been shown to improve recall performance (Carpenter \& DeLosh, 2005), as has retrieval practice of particular names (Morris et al., 2005). However, these previously studied approaches may be difficult to use in the real world; for example, one probably cannot control how many people they meet at a conference, let alone the spacing between these meetings. Semantic associations or mental imagery devices, such as creating a mnemonic around the name and associating this with a physical feature or fact about the person, have also been shown to improve how well names are remembered (e.g., McCarty, 1980). Comparisons of these techniques, however, show that mnemonic techniques are less effective than spacing (Morris et al., 2005; Neuschatz et al., 2005), and so may benefit from additional supporting cues. 


\section{MULTISENSORY ENCODING OF NAMES}

More recent research has begun to tap into the connection between the sensory content available at the time of encoding and the conditions present during later retrieval. Previous work has shown that encoding an audiovisual of a person talking is more effective for subsequent recognition of their voice than encoding the voice alone, showing the superiority of audiovisual encoding over auditory encoding in auditory recognition (von Kriegstein \& Giraud, 2006). Learned congruence between a face and voice has been reported to speed recognition of a familiar face-voice pair, compared to an incongruent audiovisual pairing (O'Mahony \& Newell, 2011). Interestingly, it has also been found that regions of the brain involved in audiovisual integration - for creating an association between congruent audio and visual cuesare activated more strongly during encoding for faces that will later be remembered than for those that are forgotten (Lee et al., 2017), so perhaps multisensory stimuli can support recall of face-name associations.

Facilitation of memory by utilizing multiple sensory cues would be consistent with a few memory models, most notably with dual-coding theory (see Clark \& Paivio, 1991; Paivio, 1991 for reviews), wherein providing verbal and non-verbal representations (that often occur across different senses) can facilitate memory. Another similar model is the cognitive theory of multimedia learning (see Mayer, 2005 for overview), wherein presentation of stimuli across verbal and pictorial working memory channels allows for better learning. In general, encoding information across different channels can provide more routes by which a memory can be accessed. This would seemingly support findings in the multisensory research literature that multisensory information can improve memory, as multisensory information provides information through at least two senses, while unisensory information can provide only one 


\section{MULTISENSORY ENCODING OF NAMES}

sensory route to a memory. However, this particular framework fails to make a distinction between having information available across multiple senses and truly multisensory experiences, where congruency (temporal, spatial, structural, semantic, etc.) between stimuli can lead to the creation of integrated multisensory representations (e.g., Spence, 2007; Laurienti et al., 2004; Lacey et al., 2007; Butler et al., 2012; Ernst \& Bülthoff, 2004; Shams \& Kim, 2010 ). The integration of cues from multiple modalities can result in overall improvement of the sensory signals, by for example, uncertainty reduction leading to improved precision and/or accuracy). We seek to investigate if multisensory mechanisms, in particular, are able to support remembering face-name associations, beyond any benefit provided by multiple unisensory traces.

Previous work indicates there may be a memory benefit to presenting stimuli with meaningful and congruent cross-modal sensory inputs (see Matusz et al., 2017; Shams \& Seitz, 2008 for an overview). For instance, studies have shown that object images are recognized better when they are originally presented with their iconic sound compared to when they are presented without sound, even when only the visual cue is presented at test (Lehmann \& Murray, 2005). Similarly, auditory recognition is better for objects originally presented together with congruent images compared to audio-alone encoding (Moran et al., 2013), or to presenting the sound with a meaningless visual stimulus (Thelen et al., 2015). Improvements to recognition memory performance were also shown to extend to written words accompanied by audio of those words (Heikkilä et al., 2015; Heikkilä \& Tiippana, 2016). While the exact mechanisms by which multisensory encoding benefits recall or recognition remain unexplained (though see proposed mechanisms in Shams \& Seitz, 2008), electroencephalographic (EEG) 


\section{MULTISENSORY ENCODING OF NAMES}

signals measured during memory retrieval begin to diverge at a relatively early stage of processing for visual versus audiovisual information (Murray et al., 2004), indicating that multisensory stimulus encoding may involve distinct processes not triggered by unisensory encoding. This would suggest that there is a distinct benefit to using multisensory cues as opposed to multiple unisensory ones, which could provide an avenue to boost memory performance in everyday tasks.

The present research seeks to expand upon these findings in a number of ways, by exploring how such mechanisms could be translated into benefitting naturalistic memories for face-name associations. Of particular note in the case of name memory, where the face and name share no semantic information, providing a visual cue that is semantically congruent with the auditory cue may prove to be beneficial. One such congruent cue that is often present at professional events but not as often studied is the name tag. Name tags, which provide visual cues that are congruent with auditory introductions, provide a natural correspondence with the spoken name and are an ideal cue for testing whether multisensory stimulus presentation can aid with associative memory tasks.

Here, in a series of experiments, we systematically investigate the role of multisensory presentations in associative memory. We present a multisensory representation of a name through the use of vocalized names and congruent name tags, to see if a multisensory stimulus presentation would aid face-name memory.

\section{Experiment 1:}




\section{MULTISENSORY ENCODING OF NAMES}

In this experiment, we examined whether name tags can improve memory of names using a within-subject design in which during the encoding phase half of the trials included a name tag and half of them did not. We hypothesized that the addition of this visual information would improve the recall of names.

\subsection{Methods}

\subsubsection{Participants}

Participants were 38 undergraduate students (22 females) at the University of California, Los Angeles. Average participant age was 19.49 years $(S D=1.07)$, and all reported normal or corrected-to-normal sight and hearing, except for one participant who reported that they did not have corrected-to-normal sight, but reported no difficulty observing the stimuli on the computer screen and were thus included in the analyses. Additionally, 30 of these participants were native English speakers. The remaining 8 were fluent in English. Initial analyses indicated that the results did not differ if non-native speakers were excluded, so those participants were kept in the analyses for this and the follow-up experiments. Two participants were excluded from analyses due to computer errors resulting in incomplete session data.

Written informed consent was obtained from each participant and experimental procedures were reviewed and approved by the UCLA Institutional Review Board.

\subsubsection{Materials}

Experimental stimuli were 60 brief video clips (1-2 s duration) of young adults (age 1822; half male, half female) captured from the chest up against a white background. In each 


\section{MULTISENSORY ENCODING OF NAMES}

video, the speaker introduced themself with the phrase "Hello, my name is [name]." Names presented during these videos were selected from the most common first names given to male and female children in the United States between 1990 and 1999 as reported by the United States Social Security Administration, so all of the names would have similar familiarity to participants.

A white rectangle acting as a name tag was placed over the chest and neck of each individual video, but did not obscure the mouth. This remained in the same location for the duration of the experiment. During half of the trials, this rectangle remained blank, presenting no additional name information to the participants (the "no tag" level of the name tag condition). In the other half of the trials, black text spelling the name given in the video was presented for the duration of the video in this white rectangle (the "tag" level of name tag condition). See Figure 1 for examples of both conditions.

Experimental stimuli were presented using PsychoPy software (Peirce et al., 2019) on a Mac Mini computer.

\subsubsection{Procedure}

The 60 videos were presented to participants across 4 blocks. During each block, participants were shown 15 videos with a mix of genders. As this meant that there were an uneven number of trials in each block, the first and third block had 8 tag trials, and the second and fourth blocks had 7 tag trials. Individuals within each block were presented in a random order, and the order of these blocks of individuals were also randomized. 


\section{MULTISENSORY ENCODING OF NAMES}

In each block, participants were first given an encoding phase (Figure 1a), where they were presented with each of the 15 videos in that block. To ensure participants were attending to the videos, they were asked to make a button-press response to report the gender of the speaker after each video, using ' 1 ' to indicate a male speaker and ' 2 ' to indicate female speaker. Participants were not informed that they would be tested later on their memory of the names. After seeing and reporting the gender for all 15 videos in the block, participants were given a 3-min break during which they were asked to close their eyes and relax. At the end of this delay, participants were given a cued recall test of the name. They were presented with a still image from each of the videos they had seen before the delay, in a randomized order, and prompted to type in the name they remembered being associated with that person. Still images were created from the final frames of each video, and were selected such that the faces had closed lips, to remove any facial cues for sounds in the name. Participants were given $10 \mathrm{~s}$ to recall and type the name; after the $10 \mathrm{~s}$, the experiment would advance to the next question.

After each cued recall attempt, participants were asked to rate their confidence in their memory for the name on a scale from 1 (low confidence) to 4 (high confidence). After being tested on all 15 names and providing confidence ratings, participants were given a 1-min break before moving on to the next block.

\subsubsection{Analysis}

Participant responses were rated by three blind raters for correctness, as well as by computer test-matching. The human- and computer-based scoring did not qualitatively alter the results, so human ratings were used to allow for spelling errors and alternative name 


\section{MULTISENSORY ENCODING OF NAMES}

spellings. Human raters were instructed to rate a response as correct if the typed response was an alternative spelling of a name, if an answer was cut off by the response time limit and could not reasonably be mistaken for another name, or if the name typed was a shortened version of the correct name that could not be mistaken for another name. If any of the raters judged a participant's attempt as correct, the response was marked as correct for final analysis. Raters largely agreed with one another, such that all three raters matched their judgments on $98 \%$ of responses.

Reaction time (RT) was collected for each key press in the typed response, and was analyzed using the first key input from the participant. As reaction times were non-normal in their distribution, median values on correct trials were used in the analyses. In the case where participants did not have any correct responses in one condition, they were removed from pairwise analyses.

\subsection{Results}

Results from the attention check (i.e. the gender judgment task) during the encoding phase showed high accuracy across all participants $(M=98.6 \%, S D=2.4 \%)$, indicating they were attending to the stimuli at encoding.

Initial analyses showed that performance differences between name tag conditions persisted across blocks, regardless of whether participants did not know their memory would be tested (as in block 1) or if they did (all subsequent blocks), so analyses collapsed performance across blocks. (Block-wise analysis of performance has been included in the supplementary materials.) Pairwise one-way t-test comparison of accuracy between the two 


\section{MULTISENSORY ENCODING OF NAMES}

conditions showed superior recall performance in the tag condition over the no-tag condition $(t(36)=3.59, p<.001$, Cohen's $d=0.42)$, such .

Pairwise one-way t-test comparison of RT for correct responses showed no significant effect of name tag condition $(t(36)=0.32, p=.75)$. The results were the same when including all trials.

a)

4)) Hello, my name

is Olivia.

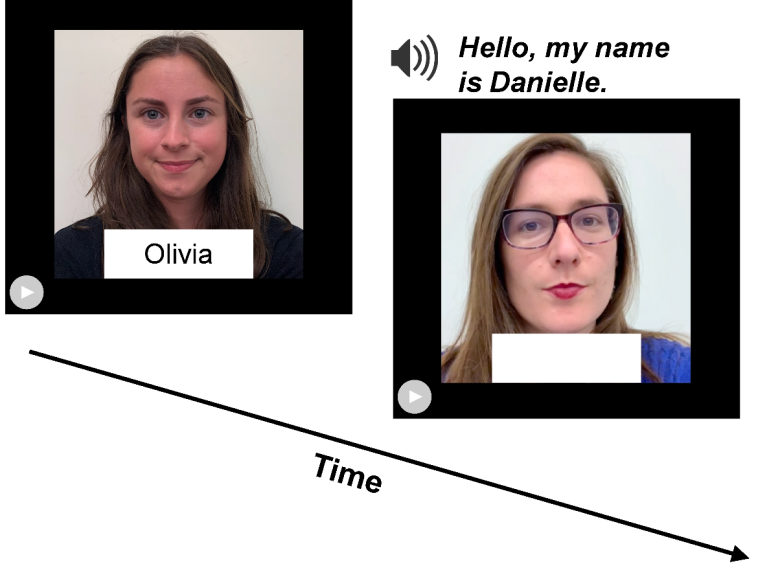

())) Hello, my name

is Kyle.

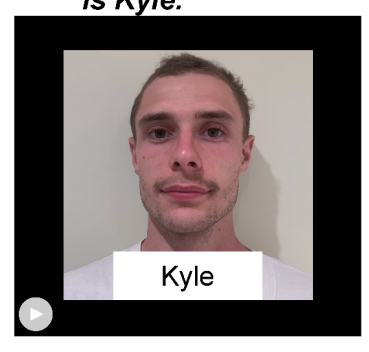

b)

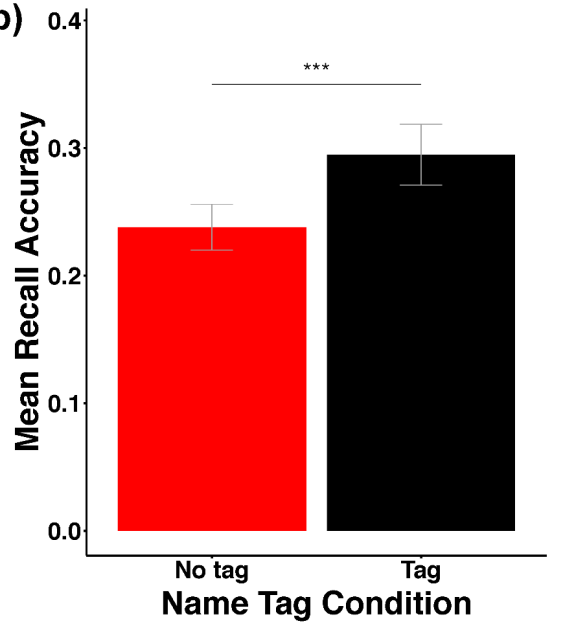

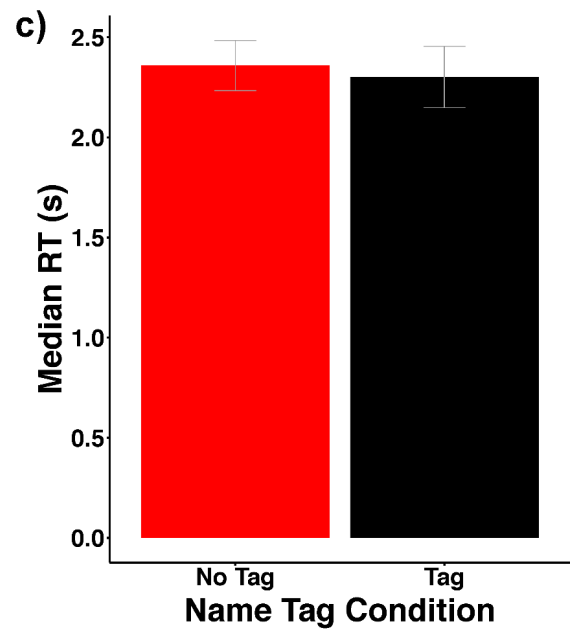

Figure 1: Experiment 1. (a) A diagram of the encoding procedure. Participants were presented with 15 videos per block, half with a name tag and half without. (b) Recall performance. There was a significant main effect such that participants recalled a higher proportion of names 
MULTISENSORY ENCODING OF NAMES

presented with a name tag compared to those presented without. (c) Reaction time (for correct responses, in seconds) for the recall task measured as the first keystroke made in the response. Error bars are standard errors.

Confidence results generally tracked the accuracy data across experiments, and can be found in the Supplementary material.

\subsection{Interim Discussion:}

Results from this experiment indicate that participants do perform better when they are given a semantic visual cue congruent with the auditory stimuli, even if these stimuli were not available at the time of retrieval. This would seem to generally support the utility of multisensory stimulus presentation for this type of recall task.

However, there are alternative explanations for the observed superiority of the tag condition. For example, It has been shown that objects presented with an accompanying irrelevant stimulus in a different modality can improve memory for that object relative to objects presented alone (Matusz et al, 2017). Alternatively, the presence of the name tag may have increased the salience of the visual stimuli, and therefore led to higher arousal in the tag condition, compared to the no tag condition that contained a blank rectangle. To investigate if the mere presence of an additional visual in the form of a name tag could explain improved performance in the tag condition, we conducted a second experiment.

\section{Experiment 2:}




\section{MULTISENSORY ENCODING OF NAMES}

In this experiment we investigated whether the superior memory performance in the previous experiment was due to the difference in visual salience in the two conditions. We compared the performance between two name tag conditions that had equal visual salience and only differed in the semantic content. In one condition, the name tag could be read and understood by the participants (English), and in the control condition it was written in a language (Armenian) that was unfamiliar and incomprehensible to the participants. If the difference in performance observed in the previous experiment was due to visual salience of the tag, then that difference should disappear in this experiment. On the other hand, if the superiority of the tag condition was due to the additional visual semantic cue, then we should observe a superior performance of the English name tag over the Armenian name tag.

\subsection{Methods}

\subsubsection{Participants}

Participants were 41 undergraduate students at the University of California, Los Angeles, with an average age of 19.66 years $(S D=1.86)$. Two participants were excluded from analyses because they knew individuals in the videos from everyday life by different names, leaving 39 participants in the analysis (30 female). All participants except one reported normal or corrected-to-normal vision, and all reported having normal hearing and being unable to read Armenian. The participant who reported not having corrected-to-normal vision reported no difficulty seeing the stimuli on the computer screen, and was kept in the analyses.

\subsubsection{Materials \& Procedure}


MULTISENSORY ENCODING OF NAMES

Videos and the name tag format matched those in experiment 1 except in the no tag condition. In this experiment, to control for the visual saliency of having a name tag, the no tag condition was replaced by a condition with a name tag written in an alphabet unfamiliar to the participants. In this half of trials, the name was written in the Armenian alphabet, so the size and shape of the letters would be similar to the names written in a familiar alphabet (see Fig. 2a), but the congruency between the visual and audio signals would not be present for participants.

The procedure and data analysis matched that of Experiment 1.

\subsection{Results}

Pairwise t-test comparison of cued recall accuracy showed superior performance in the English name-tag condition compared to the Armenian name tag condition , $t(38)=4.21, p<$ .001 , Cohen's $d=0.54$, (Fig $2 b$ ). Median reaction time for correct responses also showed an effect of tag, $t(38)=3.16, p=.003$, Cohen's $d=0.54$, such that median response time was shorter when recalling names originally presented with an English tag than an Armenian one

(Fig 2c). The results were qualitatively the same when including all trials (including incorrect responses). 
a) H)) Hello, my name
is Olivia.

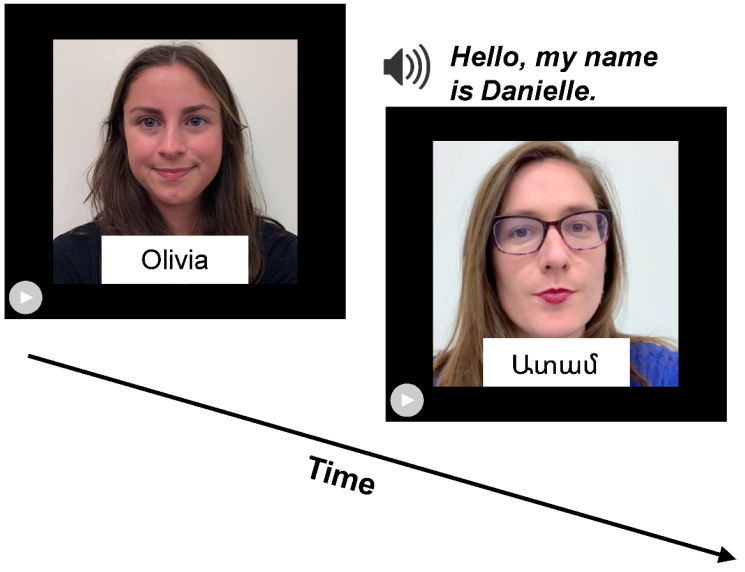

())) Hello, my name

is Kyle.

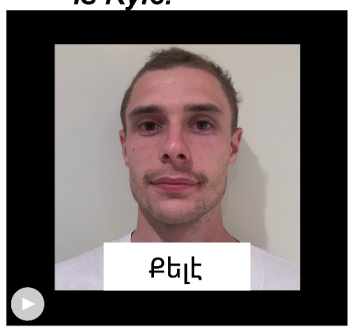

b) 0.4

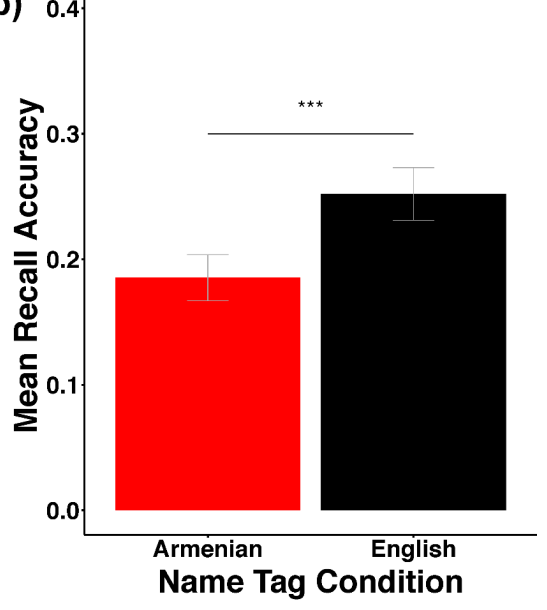

c)

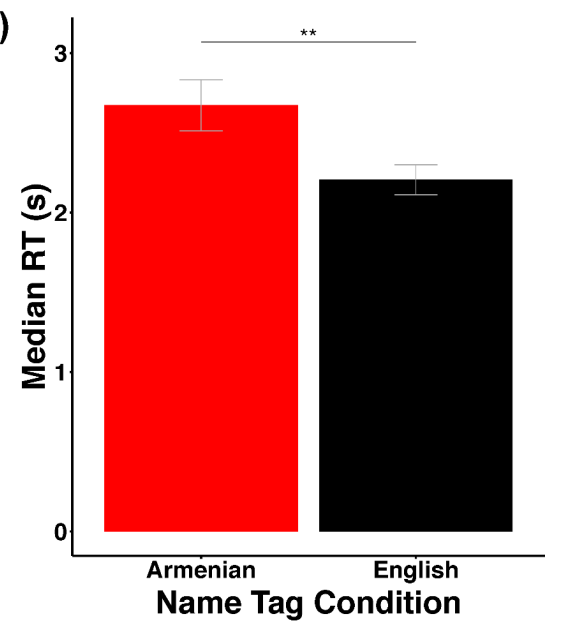

Figure 2. Experiment 2. (a) A diagram of the encoding procedure for Experiment 2. Armenian names are presented on the trials lacking a congruent name tag to control for the visual saliency of having a name tag with writing. (b) Recall performance. Participants showed significantly higher recall for names when the face was initially presented with an English name tag than with an Armenian name tag. ${ }^{* * *}=p<.001$ (c) Reaction time (for correct responses, in seconds) for the recall task measured as the first keystroke made in the response. Error bars are standard errors.

\subsection{Interim Discussion}




\section{MULTISENSORY ENCODING OF NAMES}

Results from this experiment show that semantically congruent visual stimuli can facilitate remembering names, and rules out the role of visual salience and arousal as the underlying mechanism for this facilitation. These findings are consistent with the hypothesis of multisensory integration as an underlying mechanism for improved memory.

It is important to note that in the control conditions for the previous two experiments

(no name tag or Armenian name tag) there are two cues available about the name: the acoustic cue (the voice) and the lip movement cue. Previous work has shown that lip reading may provide important multisensory cues, and can assist with disambiguating sounds (Bernstein et al., 2004). The written name (name tag) information can help encoding the face-name association in two different ways: by disambiguating (reducing the uncertainty) of the lip movement cue or by disambiguating the auditory cue. In order to gain insight into which process is occurring, we conducted the following experiment.

\section{Experiment 3:}

The goal of this experiment was to gain insight into the role of the lip movement cue in the facilitation effect of name tags observed in previous experiments. To investigate whether the observed facilitation effect stems primarily from the disambiguation of lip movements by the name tag (both visual cues, but one perceptual and the other semantic) videos were replaced by still images in this experiment, to remove lip reading cues. To the extent that the benefit of the name tag cue stems from its interaction with the lip movement cue, in this experiment the effect should disappear or be weakened. Conversely, if the benefit of name tag stems primarily from interaction with the auditory cue or just by providing an additional source 
of information without interacting with the other cues, then the effect should remain the same here.

\subsection{Methods}

\subsubsection{Participants}

A total of 44 participants ( 37 female), who were all undergraduate students at the University of California, Los Angeles, were enrolled. Participants had an average age of 19.58 years $(S D=2.11)$, and all reported normal or corrected-to-normal sight and hearing. Thirty-five reported being native English speakers, and all reported being fluent in the language. Two participants were excluded from analysis due to computer issues interrupting the experiment.

\subsubsection{Materials \& Procedure}

Materials had one major change from the preceding experiments: the video of the speaker was replaced by a still image of the individual from the video, to remove the ability of participants to use lipreading to help with the task. The images were taken from the end of each video, selected so the speakers' lips were closed and provided no cues for what sounds the individuals may have been speaking. Each image was presented for the duration of the video it was replacing, and the audio that accompanied it was taken from the original video.

The procedure and data analysis matched that of the first experiment, where the tag was either blank or had an English tag.

\subsection{Results}




\section{MULTISENSORY ENCODING OF NAMES}

Accuracy results for the recall task were very similar to those of the previous

experiments (Figure 3). Pairwise on-way t-test analyses showed higher recall for names

originally presented with a name tag compared to those presented with no tag $(t(41)=4.64, p<$ .001 , Cohen's $d=0.56)$. Median correct reaction time also showed an effect of name tag $(t(37)$

$=2.82, p=.008$, Cohen's $d=0.56)$, such that recall responses to names originally presented with a name tag were faster than those for names originally presented with no tag. The results were qualitatively the same when all reaction times were used.

a)

4)) Hello, my name

is Olivia.

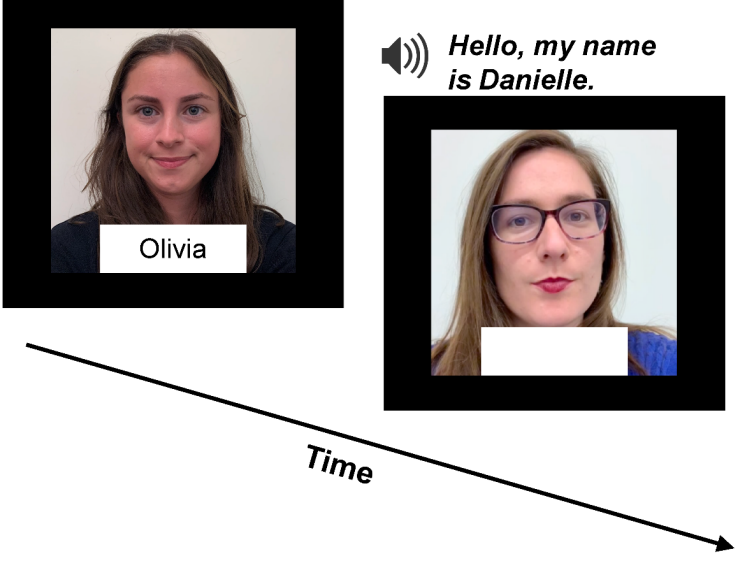

())) Hello, my name

is Kyle.

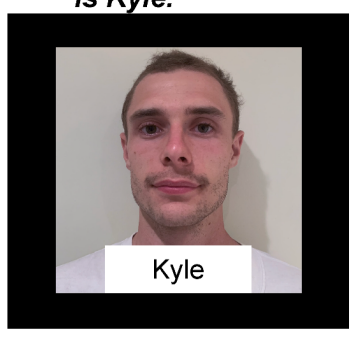

b)

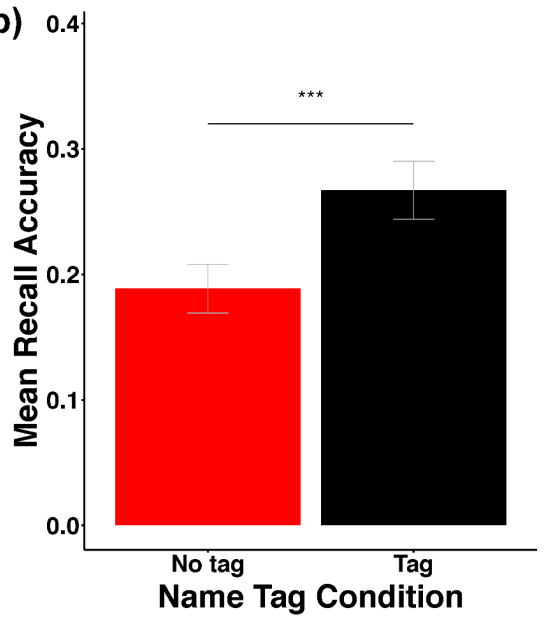

c)

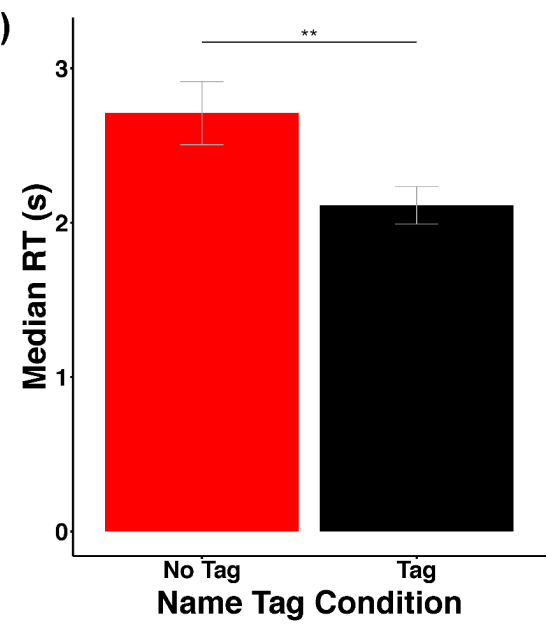


Figure 3: Experiment 3. (a) A diagram of the modified encoding procedure for Experiment 3 (still pictures instead of videos). (b) Recall performance. Participants showed higher average recall performance for names encoded with a name tag than for those encoded without. (c) Reaction time (for correct responses, in seconds) for the recall task. Measured as the first keystroke made in the response. Error bars are standard errors.

\subsection{Interim Discussion:}

Results for Experiment 3 indicate that the observed superiority of the tag condition over no tag is not due (at least entirely) to interaction with the lip-reading cue. The findings were consistent with those of experiments 1 and 2, supporting the interpretation that multisensory mechanisms may be able to explain the improved recall performance when name tags are presented at encoding. However, it should be noted that the amount of time participants were exposed to each name differed between the two conditions: when name tags were presented, participants were aware of the name much earlier than in the no tag condition. This difference in duration could lead to improved performance from longer exposure to the visual cue, rather than any multisensory mechanisms. As such, Experiment 4 was designed to keep name exposure times equal between the tag and no tag conditions.

\section{Experiment 4:}




\section{MULTISENSORY ENCODING OF NAMES}

The objective of this experiment was to equate the duration of time in which the name of the speaker is available to the participant across conditions to test whether the observed superiority of the tag condition was due to the longer duration of the name information being available in the tag condition.

\subsection{Methods}

\subsubsection{Participants}

Participants for this experiment were 49 undergraduate students ( 39 female) at the University of California, Los Angeles, with a mean age of 19.06 years $(S D=0.87)$. All participants reported normal or corrected-to-normal vision and normal hearing, and 7 reported being nonnative speakers of English, but were fluent and so kept in for analyses.

\subsubsection{Materials \& Procedure}

Videos and the name tag format matched those in Experiment 1, except all videos were cut such that the introduction ("Hello, my name is") was removed, leaving only the name. This meant that the tag and video were on screen for only the duration of the stated name.

The experimental procedure was similar to those of Experiment 1, with a few notable changes. As task performance had been, overall, somewhat low in previous experiments, the number of names participants were asked to learn per block was reduced from 15 to 10, and the number of blocks increased from 4 to 6 . Moreover, at test, the confidence rating task was replaced by a recognition memory task, to probe if recognition would benefit from multisensory encoding as well as recall. Participants were given the same 10 s to type a 


\section{MULTISENSORY ENCODING OF NAMES}

response to the recall prompt as in Experiment 1, and then given a 5-alternative multiplechoice recognition test for the name, using the same image as a prompt. The 5 names selected for the recognition test included the correct name and 4 alternatives that had been presented in the same block. To ensure this task would not be trivial and 5 probable names would exist, blocks now consisted of the same gender of speaker in all videos, resulting in 3 blocks of female and 3 blocks of male speakers. The assignment of male or female speakers to blocks was pseudorandom between participants, as was block order. As all of the speakers within one block were of the same gender, the encoding task of recognizing the gender was removed for this experiment.

\subsection{Results}

Recall results in this experiment (Fig. 4 b-c) largely follow those of the previous experiments: pairwise one-way t-test results showed that average cued recall performance was higher for names originally presented with a name tag than for names presented without a tag $(t(49)=3.11, p=.003$, Cohen's $d=0.32)$. Median correct reaction time in the recall task showed no significant effect of name tag $(t(49)=0.59, p=.56)$. Results were qualitatively the same when using all trials.

On the recognition task, there was a marginal effect of name tag condition $(t(49)=1.83$, $p=.07$, Cohen's $d=0.22$ ), such that names originally presented with a tag were remembered more often than names originally presented with no tag. There was no significant difference between name tag conditions on median correct response time in the recognition task $(t(49)=$ 
$0.75, p=.45)$. These results were qualitatively the same when analyzed using response times from both correct and incorrect trials.
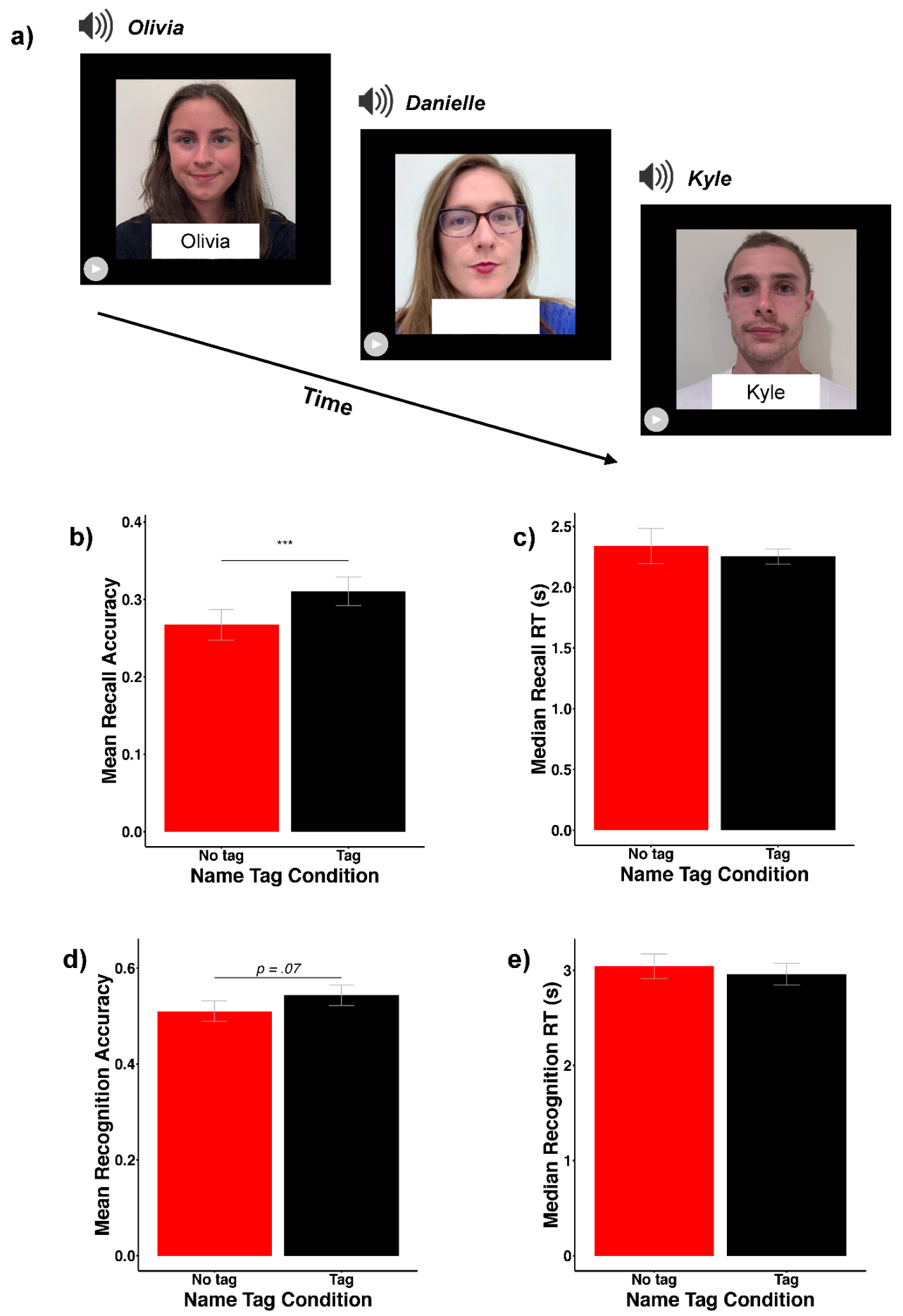
Figure 4: Experiment 4. (a) A diagram of the modified encoding procedure for Experiment 4. This design ensures the onset time, and subsequent presentation duration of the name tag and stated name are matched. (b) Recall performance. There was a main effect of name tag, such that presenting a name tag produced higher average recall than if no tag was present during encoding. (c) Reaction time (for correct responses, in seconds) for the recall task measured as the first keystroke made in the response. (e) Recognition performance. Participants showed no significant difference in recognition performance based on the tag condition. (f) Reaction time (for correct responses, in seconds) for the recognition task. Error bars are standard errors.

\subsection{Interim Discussion}

Experiment 4 further supports that the addition of a visual stimulus congruent with auditory stimulus improves performance in cued recall for names, even if the presentation of the congruent visual stimulus matches the length of the auditory stimulus. Interestingly, recognition of the names does not show a similar benefit in accuracy, though the data trends such that recognition accuracy is somewhat higher for faces originally presented with a tag compared with those that were not. 
a)

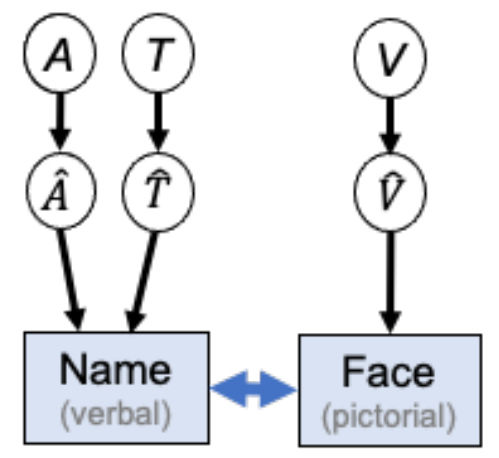

b)

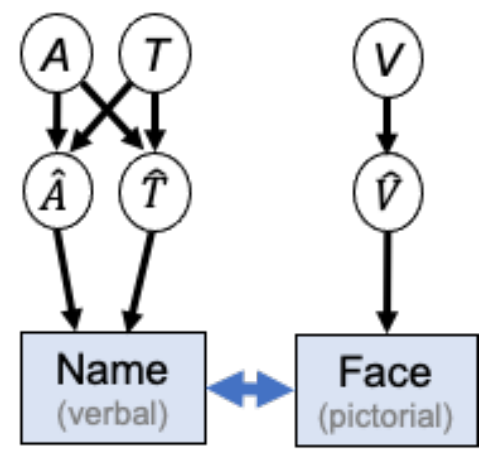

Figure 5. Two possible mechanisms underlying the name tag facilitation of face-name associative memory. $A$ denotes auditory input provided by the spoken name. $T$ denotes the visual input (text) provided by the name-tag. $V$ denotes the visual input provided by the video of the individual. A, T, and $\mathrm{V}$ are noisy sensory inputs, and $\hat{A}, \widehat{T}$, and $\widehat{V}$ represent the perceptual estimates. a) If multisensory mechanisms are not utilized, $\hat{A}$ (spoken name) and $\widehat{T}$ (written name) independently provide information about name (verbal representation), without interacting with each other perceptually. b) If multisensory mechanisms are utilized, both $\hat{A}$ and $\hat{T}$ provide information about name and each now provide an improved estimate of name due to integration with the other sensory stimulus, as depicted by arrows from both sensory stimuli to each of the perceptual estimates.

\section{Experiment 5:}

Experiments 1-4 establish that addition of a name tag improves the recall of names. However, two distinct underlying mechanisms could mediate this facilitation (see Fig. 5). One possibility is that the name tag could be serving as an additional memory trace that would aid 


\section{MULTISENSORY ENCODING OF NAMES}

recall by providing a second redundant route to the desired information (i.e. the name), Fig. 5a. Alternatively, the tag cue provides a multisensory representation of name by combining with the audio (and maybe also lip movements; Fig. 5b) and a richer encoding of name-face association. To tease apart these two potential mechanisms, in this fifth experiment we compared two conditions that were equal in the number of "traces" during encoding, but one condition allows for multisensory integration to occur, whereas the other condition does not. This was achieved by manipulating the relative timing of the cues, because it is well established that temporal congruency between cues plays an important role in integration of cues (see Calver et al, 2004; Shams \& Kim, 2010). In both conditions, the same cues (video, audio, name tag) were presented, however, in one condition the audio and tag were presented simultaneously, and in the other condition the tag followed the audio with a delay that is expected to disrupt integration. If the benefits of name tags derive exclusively from their provision of an additional memory trace, then we would expect to see equal performance across conditions. In contrast, a multisensory framework would predict that simultaneity between the audio and congruent visual stimuli would be necessary to receive a memory benefit, and therefore we should see better performance in the synchronous condition.

\subsection{Methods}

\subsubsection{Participants}

A total of 38 participants ( 24 female), who were all undergraduate students at the University of California, Los Angeles, were enrolled. Participants had a mean age of 20.89 years $(S D=3.36)$, and all reported normal or corrected-to-normal sight and hearing. Thirty-five 


\section{MULTISENSORY ENCODING OF NAMES}

reported being native English speakers, and all reported being fluent in the language. Four participants were excluded from analysis due to remembering zero names in either condition during any block of the experiment.

\subsubsection{Materials \& Procedure}

Materials were the still images from Experiment 3, as these reduce the influence of congruency between lipreading and the visual name tag from playing a role in participants performance. Still images were presented for $5.5 \mathrm{~s}$ with the audio from the original videos played starting at the visual stimulus onset. At the bottom of the image, placed over the neck and torso for the duration of the stimulus, as in experiments 1-4, was a white rectangle. Both name tag conditions in this experiment present a name tag, and differ in when the tag is displayed: synchronously with the name, or asynchronously. In the synchronous condition, the name is visible starting simultaneously with the still image and audio, and, in the asynchronous condition, the name is visible beginning $2.5 \mathrm{~s}$ after the start of the presentation of the still image. In both cases, the visual name will be presented for $2.5 \mathrm{~s}$.

Blocks are organized as in Experiment 4: a total of 6 blocks containing 10 same-gender speakers and names to remember in each block, with tests of both cued recall and recognition for the names given after a 3-min delay.

\subsection{Results}

Recall results in this experiment (Fig. 6) largely follow those of the previous experiments: a pairwise one-way t-test showed that recall performance was higher in the 
multisensory synchronous condition compared to performance in the asynchronous condition $(t(33)=2.27, p=.03$, Cohen's $d=0.22)$. There was no significant effect of name tag condition on median correct recall time, $t(32)=0.82, p=.42$. This effect was qualitatively the same when all response times were included.

Recognition results showed no significant effect of name tag condition, $t(33)=0.90, p=$ 0.37. However, there was a significant effect of name tag condition on recognition response time $(t(33)=2.27, p=.03$, Cohen's $d=0.34)$, such that participants on average had faster median responses to names originally presented synchronously than asynchronously. These results were qualitatively different when all response trials were included, such that there was no significant difference between name tag conditions when correct and incorrect responses were used $(t(33)=1.31, p=.20)$, 

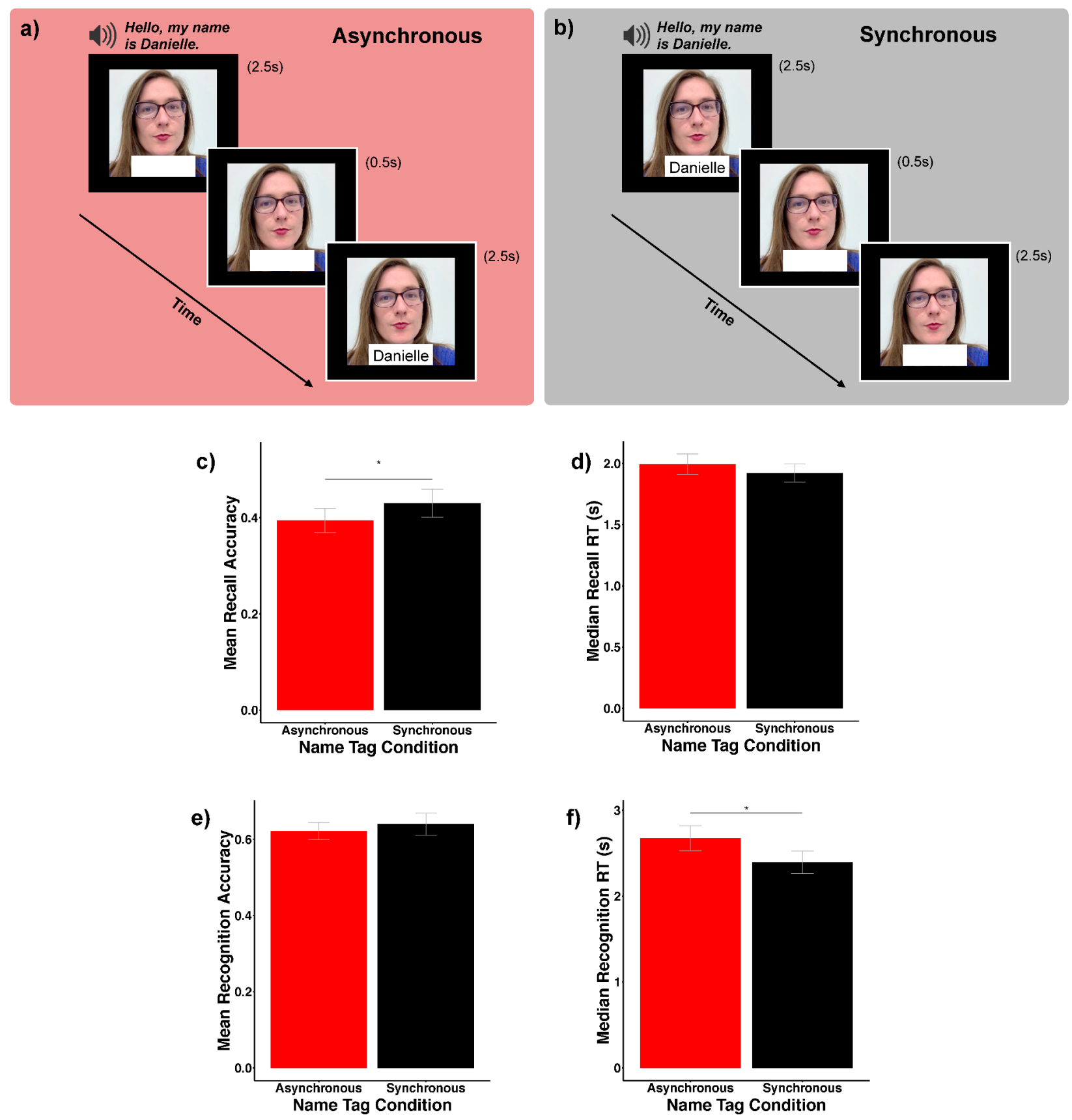

Figure 6: Experiment 5. (a) Schematic of asynchronous condition. The asynchronous condition presented the name tag after a 0.5 second delay. (b) Schematic of synchronous condition. The synchronous condition presented the name tag and audio synchronously. (c) Recall performance. There was a significant main effect of condition on recall. (d) Reaction time (for correct responses, in seconds) for the recall task, measured as the first keystroke made in the 


\section{MULTISENSORY ENCODING OF NAMES}

response. (e) Recognition performance. There was no main effect of condition on recognition. (g) Reaction time (for correct responses, in seconds) for the recognition task. Error bars are standard errors.

\section{Discussion}

In this study, we investigated memory of people's names using naturalistic stimuli of videos in which speakers introduced themselves, as is often the case in daily life. Remembering people's names in this context amounts to an associative memory task in which the brain encodes an association between a visual face/body and an auditory presentation of a name (although the lip movements of the speaker may also contribute to this encoding). Because there is no inherent relationship between one's name and one's face, the learning and retention of this association is non-trivial, especially when tasked with the learning of multiple face-name pairs within a short period of time, which is often the case when we attend a party or a professional event.

A few previous studies have shown that multisensory encoding of objects or object features (e.g., motion, or voice) facilitates learning (e.g., Seitz et al, 2006; von Kriegstein \& Giraud, 2006; Kim et al, 2008; Shams et al., 2011) and episodic memory (e.g., Lehman \& Murray, 2005; Moran et al, 2013; Heikkilä et al., 2015). However, these learning and memory tasks involved processing of a single feature or recognition of an object or object feature, and did not involve memory of an association. Here, we examined whether the benefit of multisensory encoding extends to associative memory. Specifically, we asked whether a multisensory encoding of a name can aid people's ability to bind that name to a face. To render 


\section{MULTISENSORY ENCODING OF NAMES}

the encoding of a name multisensory, we added a written representation of the name in the form of a name tag in addition to the auditory introduction given by the speaker. We then compared the memory of names in the presence and absence of name tags.

Across a series of five experiments, we found that participants, when cued with a face, were more likely to remember the associated name when that name had been encoded with a name tag, compared to when no name tag was provided. Experiment 1 showed a robust superiority of the tag condition (effect size 0.42 ) over the no tag condition. Experiment 2 examined whether the observed effect in Experiment 1 was due to the difference in visual saliency of the two conditions (blank vs. text below the face) by controlling for the visual saliency. In both conditions name tags were presented, but in one condition they were in English and congruent with the spoken name, and in the other condition they were in Armenian (a language that could not be understood by participants) and not congruent with the spoken English name. The English tag condition resulted in superior cued recall performance compared to the unintelligible name tag (effect size 0.54), ruling out that the difference in performance was due to visual saliency. Experiment 4 examined whether the observed effect in the earlier experiments was due to the fact that name information was available to the observers throughout the trial whereas the name information conveyed by voice was only available for a portion of trial duration. In that experiment, the presentation of the name tag during the trial was cut and matched the duration of the vocalization of the name. The name tag advantage effect persisted, ruling out the role of the difference in duration of name information as the underlying factor. These experiments collectively establish that the presentation of name tag aids memory of names by providing an additional cue for name. However the mechanism by 


\section{MULTISENSORY ENCODING OF NAMES}

which this additional cue facilitates face-name memory remains unclear. Experiments 3 and 5 aimed to shed light on this question.

The name tag cue is a visual semantic cue. It can interact and disambiguate (reduce the uncertainty of) the other semantic cues, namely the vocal cue and the lip-reading cue. The lip reading cue is an impoverished cue and, as such, could benefit from disambiguation in a withinmodality (vision) manner when a name tag is added, bypassing multisensory mechanisms. Therefore, we asked if the interaction between name tag and the lip movements is the primary factor underlying the observed facilitation of memory. In Experiment 3, lip movement cues were eliminated by replacing videos with static images during the encoding phase. The superiority of name tag condition over no-name tag persisted with a similar effect size (effect size 0.26 with the lip movement vs. 0.34 without lip movements), suggesting that the putative enhancement of lip reading cue by name tag cannot account for the observed effect.

Finally, we aimed to gain insight into the underlying mechanism of the name tag benefit by teasing apart the role of multiple independent memory traces (Fig. 5a) vs. the role of integration of multisensory cues (Fig. 5b). The name tag provides an additional memory trace, which can facilitate recall by providing an alternative retrieval route to access the name when cued with the face. That is, the face might trigger the retrieval of the auditory memory of the spoken name or the visual memory of the written name, essentially giving participants an extra chance to succeed at recalling the name. In this framework (Fig. 5a), the mere existence of an additional cue is sufficient for improved recall. On the other hand, in the multisensory encoding framework (Fig. 5b), the interaction between the cues and the integrated representation of the feature/object can play a key role in the richness of the encoding, thus increasing the likelihood 


\section{MULTISENSORY ENCODING OF NAMES}

of later recall (Shams \& Seitz, 2008). More specifically, the name tag cue can be integrated with the vocal cue, resulting in a more accurate and/or more precise representation of the name. This improved name representation can strengthen the encoding of the face-name association and lead to improved memory performance.

In order to tease apart these two possible accounts, in Experiment 5, we compared two conditions in which the number of traces were equivalent, but one condition lends itself to integration of the name tag cue with other cues, whereas the other condition does not. It is well established that temporal congruency is key in integration of sensory cues, and the lower the temporal congruency the lower the probability of integration (e.g., Shams et al., 2002; Shams \& Kim, 2010; Calvert et al., 2004; see Ernst \& Bülthoff, 2004). Therefore, by manipulating the relative timing of the name tag and the vocal (and lip movement) cues, we can influence their probability of integration. It has been shown that introducing audio and visual stimulus onset asynchronies of between 150 and 250 ms reduces audiovisual speech fusion and alters brain activity in speech-processing regions of the brain (Macaluso et al., 2004; van Atteveldt et al., 2007; Miller \& D’Esposito, 2005). Therefore, it is to be expected that the name tag cue would get integrated with the other name cues when it is presented synchronously and not integrated when it is presented with a delay of $500 \mathrm{~ms}$. On the other hand, in both of these conditions all of the cues are available in each trial, and by delaying the name tag relative to the video, the performance may even be expected to improve according to multiple independent memory trace account: the name information which is initially encoded by voice and lipreading, gets reinforced by the later presentation of the name tag. The results of Experiment 5 showed that the synchronous presentation of the name tag leads to better memory 


\section{MULTISENSORY ENCODING OF NAMES}

performance than the asynchronous presentation. This would support the multisensory integration hypothesis, that multisensory object representation itself can be helpful to memory above what would be predicted by having multiple independent sensory traces. Future research will need to probe this question further by examining the nature of multisensory interactions that promote facilitation of memory, including which sensory combinations can facilitate memory performance, and what kinds of memory tasks will benefit from multisensory integration.

The present results cannot be accounted for by the dual-coding theory or the cognitive theory of multimedia learning, according to which the combined verbal and pictorial presentation of words facilitates memory compared to verbal-alone presentations. In the present study, in all conditions, including the baseline no tag condition both verbal (name) and non-verbal (video/image) representations are available (see Fig. 5). The only difference between the experimental and control conditions is the availability of additional verbal information (name tag), or, in the case of Experiment 5, the relative timing of the additional verbal (name tag) information.

The improved memory accuracy under multisensory stimulus presentation conditions does not seem to be as robust in the multiple-choice recognition task compared to the recall task. Experiments 4 and 5 evaluated both name recall and name recognition in response to face cues. In Experiment 4 there was a trend for a multisensory benefit in recognition, whereas in both experiments the benefit of multisensory presentation in recall was statistically significant. While previous experiments have shown multisensory benefits in recognition tasks, those experiments were structured quite differently from the current experiment. This 


\section{MULTISENSORY ENCODING OF NAMES}

experiment, unlike many previous multisensory memory studies, used an associative memory task. Previous multisensory research has probed memory for single items, while the current study investigated memory for an association between a name and a face. Moreover, additional experimental power may be needed to uncover statistically significant effect in the recognition task.

Also of note are that the brain mechanisms by which multisensory stimuli benefit recall performance are unclear. The current results can speak to a few different behavioral theories, but cannot distinguish between them decisively. Previous work has indicated that cross-modal interactions allow for information distributed across multiple senses to be combined into meaningful representations. This combination of senses has been found to allow for optimal processing of sensory information and can help disambiguate noisy stimulus presentation via uncertainty reduction (one signal can disambiguate another signals, leading to the improvement in precision and/or accuracy) (see Ernst \& Bülthoff, 2004; Shams \& Beierholm, 2010 for overviews). Multisensory stimulus presentation may also change how attention is directed and multisensory scenes are segregated (Lewkowicz et al, 2021) at the time of encoding. Which mechanism, if any of these, supports the current findings is currently unclear, and future neuroimaging research may be able to identify the neural mechanisms supporting multisensory memory benefits.

Our findings are generally in line with previous multisensory findings, and expand those results to associative memory, and to a more naturalistic memory task. The current experiments also suggest that multisensory mechanisms can be leveraged in daily, difficult tasks to improve memory performance. These findings do not contradict previous memory 
theories, but rather can function as an additional tool that can be used to improve human memory in difficult situations. Traditional techniques for improving memory-including mnemonics and spatial mapping-are effective, but do require a relatively high level of sophistication and intent to employ. Using basic sensory information could be more easily and passively implemented to improve memory. This could lead to the development of new strategies, techniques, and technologies to improve everyday life and learning, even for relatively difficult associative memory tasks. 
MULTISENSORY ENCODING OF NAMES

\section{Acknowledgements}

We would like to thank Ashkan Arjomandi, Fatima Iqbal, Chenyang Lin, Golbarg Rahimi, Abheri Setlur, and Sarah Thraen for rating participant responses.

\section{Declarations:}

Funding: This work was supported by the UCLA Faculty Senate intramural grant.

Conflict of interest: The authors have no relevant financial or non-financial interests to disclose.

Ethics approval: The experiments in this manuscript were approved by

the University of California, Los Angeles Institutional Review Board in accordance with the Declaration of Helsinki (IRB\# 18-002000).

Consent to participate: Informed consent was obtained from all individual participants included in the study.

Consent for publication: The authors affirm that human research participants provided informed consent for publication of the images in Figures 1-4 and 6. (Participants recorded for the stimuli did not provide consent for their pictures to be used in publication. Thus, Figures 1-4 and 6 use other individuals staged to resemble the experimental stimuli who did consent for their images to be used.)

Availability of Data and Materials: Participant data has been made available at https://github.com/murray-carolynA/Data_MultisensoryNametagStudy. For access to stimuli, please contact Carolyn Murray (camurray@ucla.edu)

Code Availability: Analysis code will be available upon request. Please contact Carolyn Murray (camurray@ucla.edu) 
MULTISENSORY ENCODING OF NAMES

\section{Open Practices Statement:}

The data for these studies are available at https://github.com/murray-

carolynA/Data_MultisensoryNametagStudy, and all other materials may be obtained upon request. None of the experiments were preregistered. 


\section{MULTISENSORY ENCODING OF NAMES}

\section{References}

Bernstein, L. E., Auer, E. T., \& Takayanagi, S. (2004). Auditory speech detection in noise enhanced by lipreading. Speech Communication, 44(1), 5-18. https://doi.org/10.1016/j.specom.2004.10.011

Brooks, J. O., Friedman, L., Gibson, J. M., \& Yesavage, J. A. (1993). Spontaneous mnemonic strategies used by older and younger adults to remember proper names. Memory, 1(4), 393-407. https://doi.org/10.1080/09658219308258245

Butler, J. S., Foxe, J. J., Fiebelkorn, I. C., Mercier, M. R., \& Molholm, S. (2012). Multisensory Representation of Frequency across Audition and Touch: High Density Electrical Mapping Reveals Early Sensory-Perceptual Coupling. Journal of Neuroscience, 32(44), 15338-15344. https://doi.org/10.1523/JNEUROSCI.1796-12.2012

Calvert, G. A., \& Thesen, T. (2004). Multisensory integration: Methodological approaches and emerging principles in the human brain. Journal of Physiology-Paris, 98(1), 191-205. https://doi.org/10.1016/j.jphysparis.2004.03.018

Carpenter, S. K., \& DeLosh, E. L. (2005). Application of the testing and spacing effects to name learning. Applied Cognitive Psychology, 19(5), 619-636.

https://doi.org/10.1002/acp.1101

Clark, J. M., \& Paivio, A. (1991). Dual coding theory and education. Educational Psychology Review, 3(3), 149-210. https://doi.org/10.1007/BF01320076

Cohen, G., \& Faulkner, D. (1986). Memory for proper names: Age differences in retrieval. British Journal of Developmental Psychology, 4(2), 187-197. https://doi.org/10.1111/j.2044835X.1986.tb01010.x 


\section{MULTISENSORY ENCODING OF NAMES}

Ernst, M. O., \& Bülthoff, H. H. (2004). Merging the senses into a robust percept. Trends in Cognitive Sciences, 8(4), 162-169. https://doi.org/10.1016/j.tics.2004.02.002

Heikkilä, J., Alho, K., Hyvönen, H., \& Tiippana, K. (2015). Audiovisual Semantic Congruency During Encoding Enhances Memory Performance. Experimental Psychology, 62(2), 123130. https://doi.org/10.1027/1618-3169/a000279

Heikkilä, J., \& Tiippana, K. (2016). School-aged children can benefit from audiovisual semantic congruency during memory encoding. Experimental Brain Research, 234(5), 1199-1207. https://doi.org/10.1007/s00221-015-4341-6

Kim, R. S., Seitz, A. R., \& Shams, L. (2008). Benefits of Stimulus Congruency for Multisensory Facilitation of Visual Learning. PLOS ONE, 3(1), e1532. https://doi.org/10.1371/journal.pone.0001532

Lacey, S., Tal, N., Amedi, A., \& Sathian, K. (2009). A Putative Model of Multisensory Object Representation. Brain Topography, 21(3), 269-274. https://doi.org/10.1007/s10548009-0087-4

Laurienti, P. J., Perrault, T. J., Stanford, T. R., Wallace, M. T., \& Stein, B. E. (2005). On the use of superadditivity as a metric for characterizing multisensory integration in functional neuroimaging studies. Experimental Brain Research, 166(3), 289-297. https://doi.org/10.1007/s00221-005-2370-2

Lee, H., Stirnberg, R., Stöcker, T., \& Axmacher, N. (2017). Audiovisual integration supports facename associative memory formation. Cognitive Neuroscience, 8(4), 177-192. https://doi.org/10.1080/17588928.2017.1327426 


\section{MULTISENSORY ENCODING OF NAMES}

Lehmann, S., \& Murray, M. M. (2005). The role of multisensory memories in unisensory object discrimination. Cognitive Brain Research, 24(2), 326-334. https://doi.org/10.1016/j.cogbrainres.2005.02.005

Lewkowicz, D. J., Schmuckler, M., \& Agrawal, V. (2021). The multisensory cocktail party problem in adults: Perceptual segregation of talking faces on the basis of audiovisual temporal synchrony. Cognition, 214, 104743. https://doi.org/10.1016/j.cognition.2021.104743

Macaluso, E., George, N., Dolan, R., Spence, C., \& Driver, J. (2004). Spatial and temporal factors during processing of audiovisual speech: A PET study. Neurolmage, 21(2), 725-732. https://doi.org/10.1016/j.neuroimage.2003.09.049

Matusz, P. J., Wallace, M. T., \& Murray, M. M. (2017). A multisensory perspective on object memory. Neuropsychologia, 105, 243-252.

https://doi.org/10.1016/j.neuropsychologia.2017.04.008

Mayer, R. E. (2014). Cognitive Theory of Multimedia Learning. In R. E. Mayer (Ed.), The Cambridge Handbook of Multimedia Learning (2nd ed., pp. 43-71). Cambridge University Press. https://doi.org/10.1017/СBO9781139547369.005

McCarty, D. L. (1980). Investigation of a visual imagery mnemonic device for acquiring facename associations. Journal of Experimental Psychology: Human Learning and Memory, 6(2), 145-155. https://doi.org/10.1037/0278-7393.6.2.145

McWeeny, K. H., Young, A. W., Hay, D. C., \& Ellis, A. W. (1987). Putting names to faces. British Journal of Psychology, 78(2), 143-149. https://doi.org/10.1111/j.20448295.1987.tb02235.x 


\section{MULTISENSORY ENCODING OF NAMES}

Miller, L. M., \& D’Esposito, M. (2005). Perceptual Fusion and Stimulus Coincidence in the CrossModal Integration of Speech. Journal of Neuroscience, 25(25), 5884-5893. https://doi.org/10.1523/JNEUROSCI.0896-05.2005

Moran, Z. D., Bachman, P., Pham, P., Hah Cho, S., Cannon, T. D., \& Shams, L. (2013). Multisensory Encoding Improves Auditory Recognition. Multisensory Research, 26(6), 581-592. https://doi.org/10.1163/22134808-00002436

Morris, P. E., Fritz, C. O., Jackson, L., Nichol, E., \& Roberts, E. (2005). Strategies for learning proper names: Expanding retrieval practice, meaning and imagery. Applied Cognitive Psychology, 19(6), 779-798. https://doi.org/10.1002/acp.1115

Murray, M. M., Michel, C. M., Grave de Peralta, R., Ortigue, S., Brunet, D., Gonzalez Andino, S., \& Schnider, A. (2004). Rapid discrimination of visual and multisensory memories revealed by electrical neuroimaging. Neurolmage, 21(1), 125-135. https://doi.org/10.1016/j.neuroimage.2003.09.035

Neuschatz, J. S., Preston, E. L., Toglia, M. P., \& Neuschatz, J. S. (2005). Comparison of the Efficacy of Two Name-Learning Techniques: Expanding Rehearsal and Name-Face Imagery. The American Journal of Psychology, 118(1), 79-102. JSTOR.

O'Mahony, C., \& Newell, F. N. (2012). Integration of faces and voices, but not faces and names, in person recognition. British Journal of Psychology, 103(1), 73-82. https://doi.org/10.1111/j.2044-8295.2011.02044.x

Paivio, A. (1991). Dual coding theory: Retrospect and current status. Canadian Journal of Psychology/Revue Canadienne de Psychologie, 45(3), 255-287. http://dx.doi.org/10.1037/h0084295 


\section{MULTISENSORY ENCODING OF NAMES}

Peirce, J., Gray, J. R., Simpson, S., MacAskill, M., Höchenberger, R., Sogo, H., Kastman, E., \& Lindeløv, J. K. (2019). PsychoPy2: Experiments in behavior made easy. Behavior Research Methods, 51(1), 195-203. https://doi.org/10.3758/s13428-018-01193-y

Seitz, A. R., Kim, R., \& Shams, L. (2006). Sound Facilitates Visual Learning. Current Biology, 16(14), 1422-1427. https://doi.org/10.1016/j.cub.2006.05.048

Shams, L., \& Beierholm, U. R. (2010). Causal inference in perception. Trends in Cognitive Sciences, 14(9), 425-432. https://doi.org/10.1016/j.tics.2010.07.001

Shams, L., Kamitani, Y., \& Shimojo, S. (2002). Visual illusion induced by sound. Cognitive Brain Research, 14(1), 147-152. https://doi.org/10.1016/S0926-6410(02)00069-1

Shams, L., \& Kim, R. (2010). Crossmodal influences on visual perception. Physics of Life Reviews, 7(3), 269-284. https://doi.org/10.1016/j.plrev.2010.04.006

Shams, L., \& Seitz, A. R. (2008). Benefits of multisensory learning. Trends in Cognitive Sciences, 12(11), 411-417. https://doi.org/10.1016/j.tics.2008.07.006

Shams, L., Wozny, D. R., Kim, R. S., \& Seitz, A. (2011). Influences of Multisensory Experience on Subsequent Unisensory Processing. Frontiers in Psychology, 2. https://doi.org/10.3389/fpsyg.2011.00264

Spence, C. (2007). Audiovisual multisensory integration. Acoustical Science and Technology, 28(2), 61-70. https://doi.org/10.1250/ast.28.61

Thelen, A., Talsma, D., \& Murray, M. M. (2015). Single-trial multisensory memories affect later auditory and visual object discrimination. Cognition, 138, 148-160. https://doi.org/10.1016/j.cognition.2015.02.003 
Tukey, J. W. (1949). Comparing Individual Means in the Analysis of Variance. Biometrics, 5(2), 99-114. JSTOR. https://doi.org/10.2307/3001913

van Atteveldt, N. M., Formisano, E., Blomert, L., \& Goebel, R. (2007). The Effect of Temporal Asynchrony on the Multisensory Integration of Letters and Speech Sounds. Cerebral Cortex, 17(4), 962-974. https://doi.org/10.1093/cercor/bhl007

von Kriegstein, K., \& Giraud, A.-L. (2006). Implicit Multisensory Associations Influence Voice Recognition. PLoS Biology, 4(10), e326. https://doi.org/10.1371/journal.pbio.0040326 
MULTISENSORY ENCODING OF NAMES

\section{Supplementary material}

\section{Confidence rating results}

\section{Experiment 1:}

Confidence ratings were analyzed using only trials where participants recalled the name correctly. That resulted in 25 of our 37 participants having missing confidence scores in at least one condition and block, so only pairwise comparisons were run on the data, to maintain as much power as possible in the analyses. A Tukey's HSD test showed no significant difference in confidence ratings across the name tag condition $(q=0.08, p=.49)$. Results of the Tukey's test for block showed that block 1 had lower confidence than all of the other blocks ( $p \leq .002$ for all comparisons with block 1), though no other blocks significantly differed from one another ( $p \geq$ .84 for all comparisons; Fig. S1). 


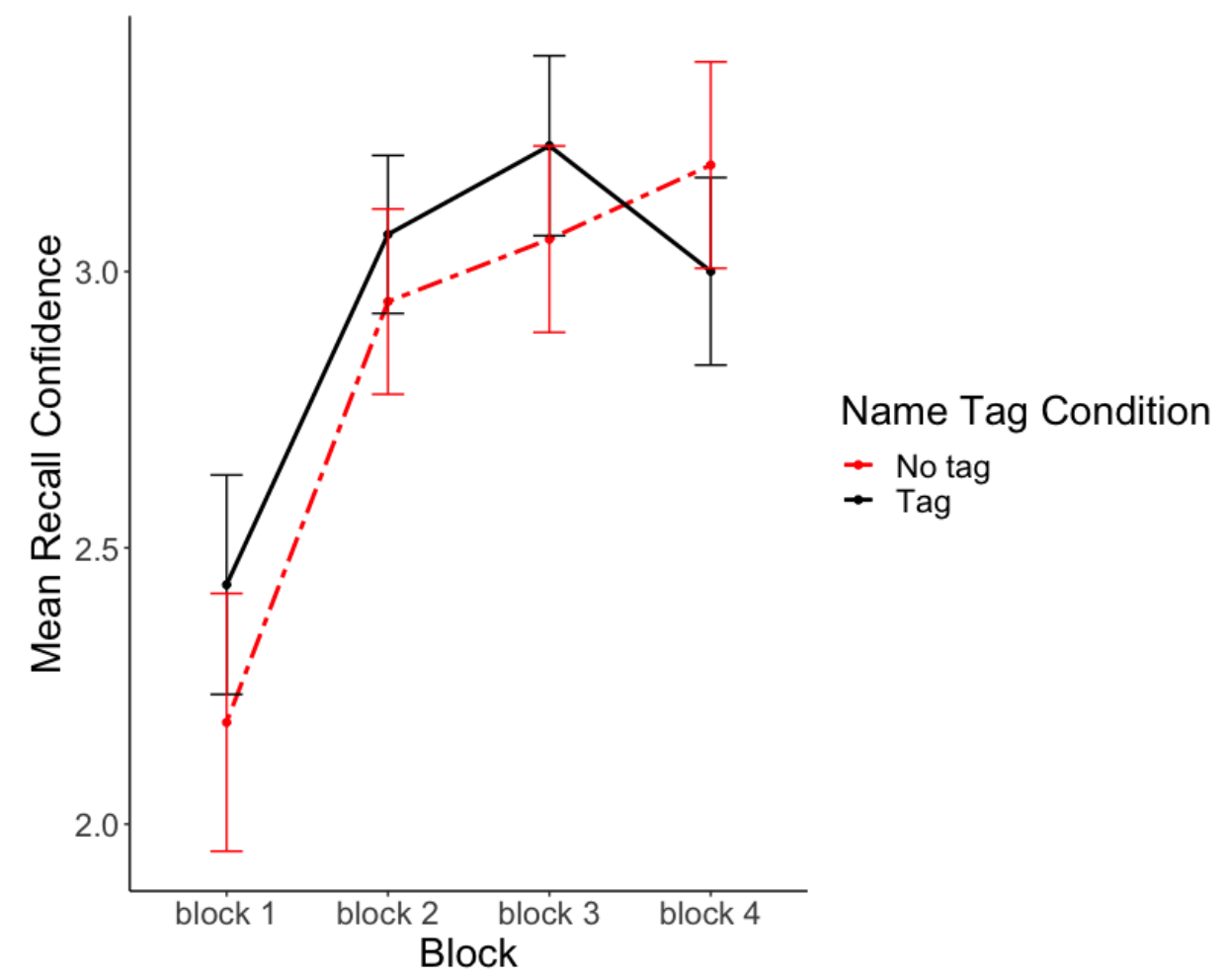

Figure S1: Average Confidence Ratings Across Blocks. Average confidence ratings assessed after recall task for each participant. Confidence averages were calculated from trials where name recall was correct. There is no significant effect of name tag condition, but there is a significant effect of block, such that block 1 confidence ratings were significantly lower than those of all other blocks.

\section{Experiment 2:}

Confidence ratings followed a similar trend to accuracy (Fig. S2), where there was a trending main effect of the name tag condition such that participants were more confident in their correct recall for names originally presented with English tags compared to Armenian tags ( $q=$ $0.22, p=.08)$. There was also a significant effect of blocks, such that confidence scores on block 
1 were lower than those of blocks 3 and 4 ( $p \leq .001$ for each comparison), and marginally lower than those of block $2(p=.06)$.

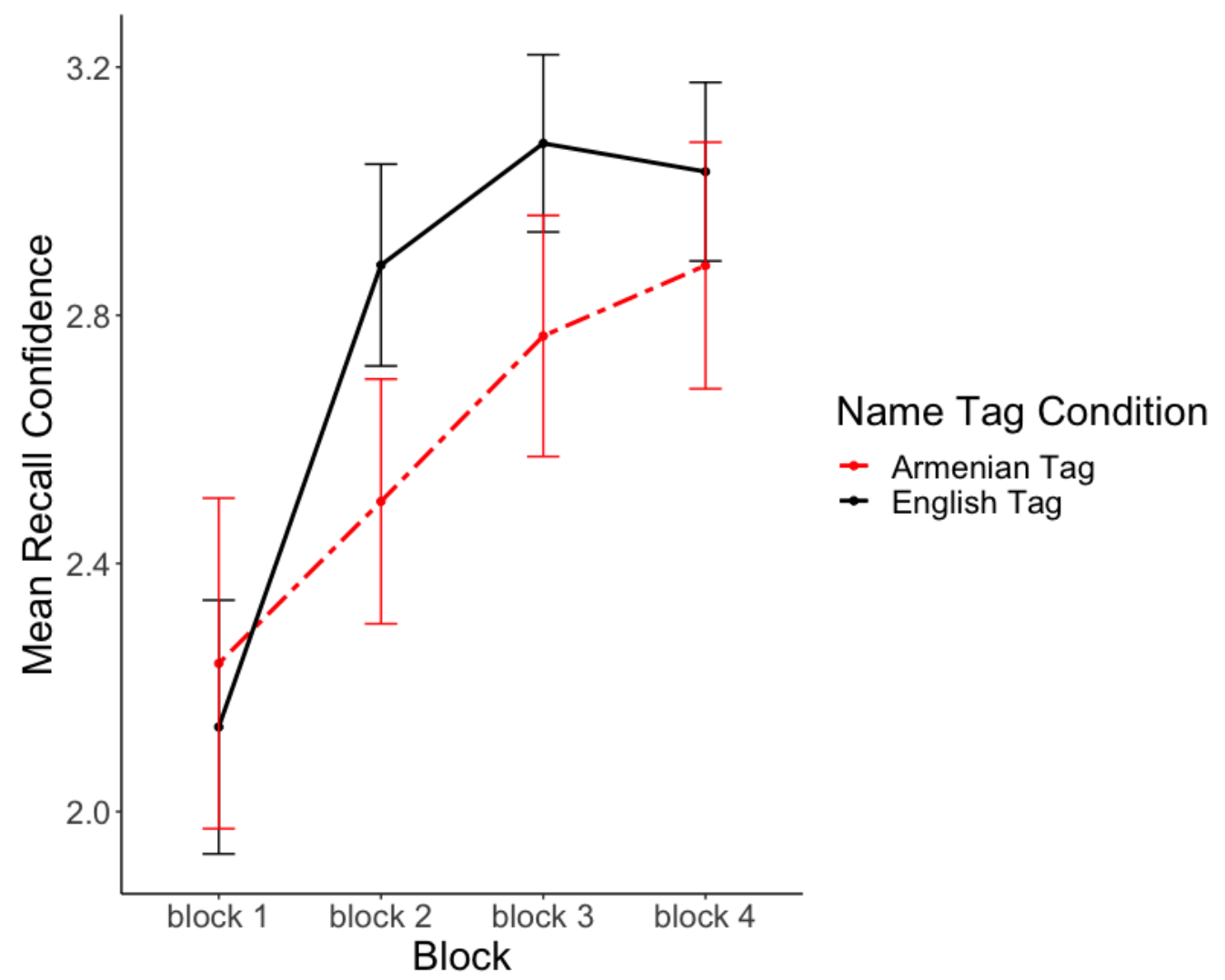

Figure S2: Average Confidence Ratings Across Blocks. Average confidence ratings assessed after recall task for each participant. Confidence averages were calculated from trials where name recall was correct. There was a trend towards English tags having higher confidence ratings than Armenian tags, and a significant effect of block, such that block 1 confidence ratings were significantly lower than blocks 3 and 4, and trended towards being lower than block 2.

\section{Experiment 3:}


Confidence ratings in the still-image experiment showed a significant effect of the name tag condition such that participants reported higher confidence in correct recall responses when the faces had been originally paired with a name tag compared to those that had not $(q=$ $0.42, p<.001 ;$ Fig. S3). Block-wise comparisons showed that confidence ratings on block 1 were significantly lower than those of block $4(p=.04)$, though no other blocks differed significantly from one another ( $p \geq .28$ for all comparisons).

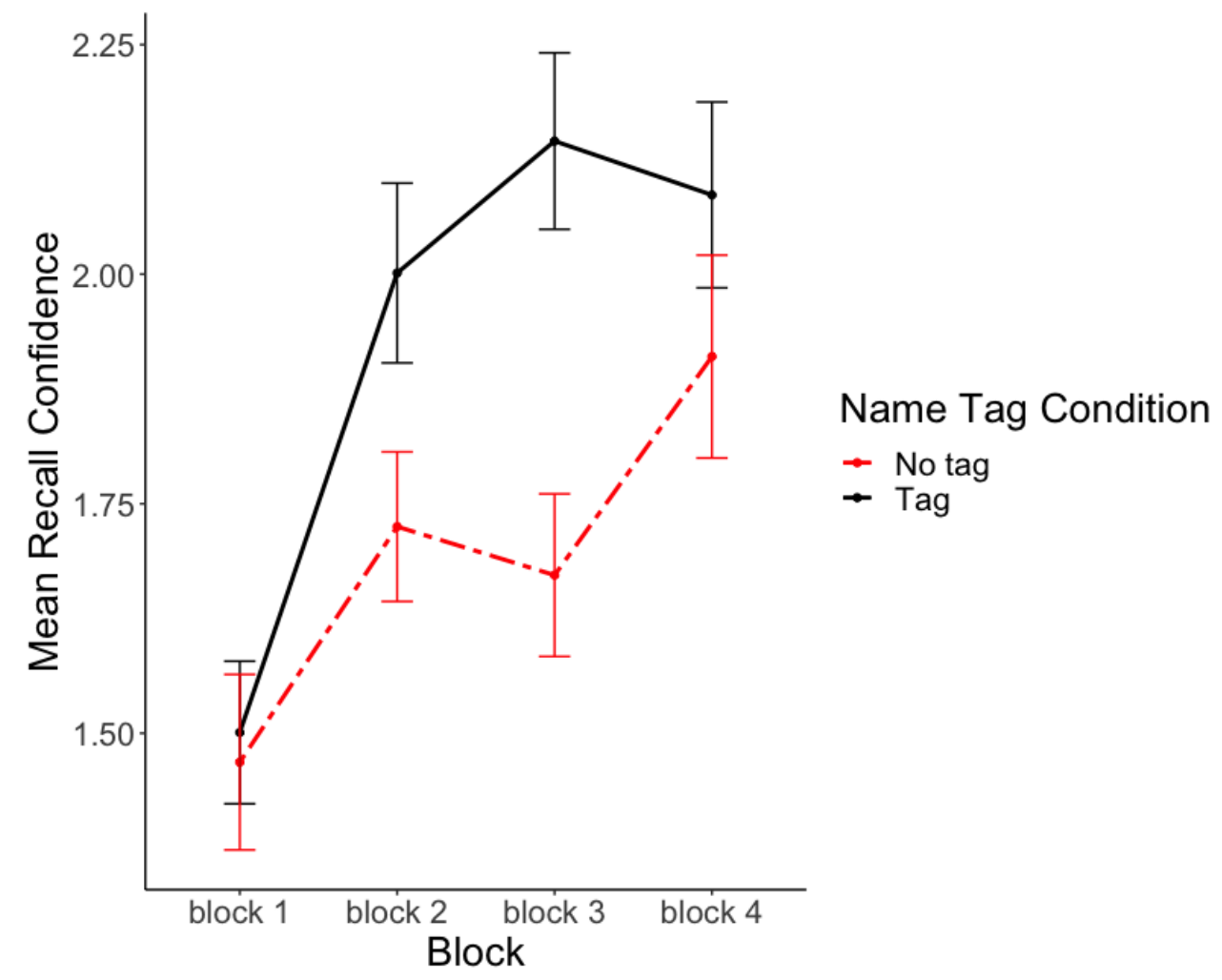

Figure S3: Average Confidence Ratings Across Blocks. Average confidence ratings assessed after recall task for each participant. Confidence averages were calculated from trials where name recall was correct. Participants were significantly more confident in their recall performance for names previously shown with a tag compared to those encoded without a tag, and confidence scores given in block 1 were significantly less than those given in block 4 . 


\section{Block-wise Results}

\section{Experiment 1:}

Accuracy results in the cued recall task were analyzed using a $2 \times 4$ repeated measures ANOVA, to assess differences between the tag and no tag conditions across all 4 blocks (Fig. S4a). There was a main effect of tag such that, on average, participants recalled more names for people who had been encoded with a name tag compared to those without one, $F(1,36)=$ 13.18, $p<.001$, partial $\eta^{2}=0.27$. There was also a main effect of block, $F(3,108)=16.55, p<$ .001 , partial $\eta^{2}=0.31$. To investigate this further, and compare performance in all possible pairs of blocks, a Tukey's HSD test (Tukey, 1949) was run to investigate block differences. This test revealed that performance in block 1 was significantly lower than other blocks $(p<.001$ for all block 1 comparisons), though there were no significant differences between these other blocks ( $p=.44$ or larger for all between-block comparisons). This very likely reflects that participants were generally unaware they would be asked to remember names during block 1 , but knew they would be tested on them in the ensuing blocks. There was no significant interaction of the name tag condition with block, $F(3,108)=0.70, p=.55$.

As RT distributions were non-normal, median RT values were used in the analysis (Fig S4b). These analyses were run on all RTs, regardless of whether the response was correct or incorrect for the trial. There was no significant difference in RT between the two tag conditions, $F(1,36)=0.13, p=.72$, or between blocks, $F(3,108)=1.41, p=0.13$. There was also no significant interaction between blocks and tag condition, $F(3,108)=1.61, p=.19$ 
a)

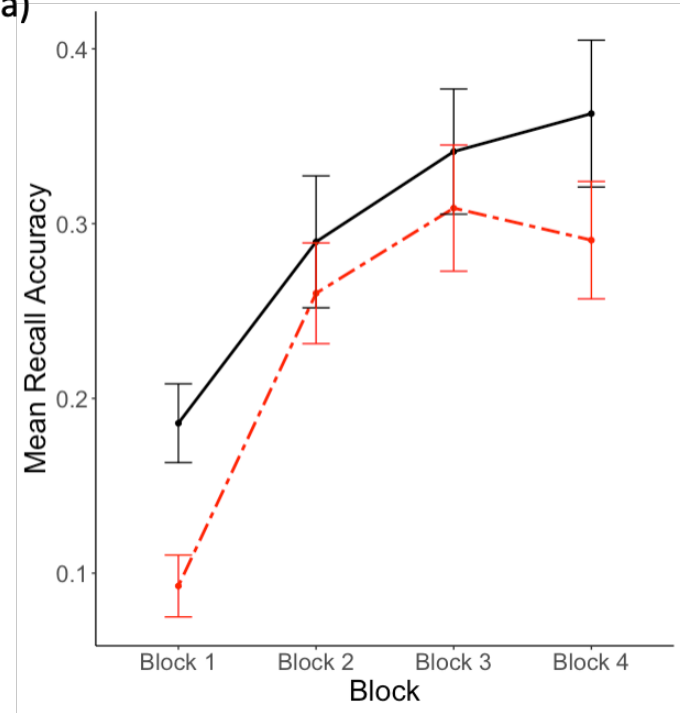

b)

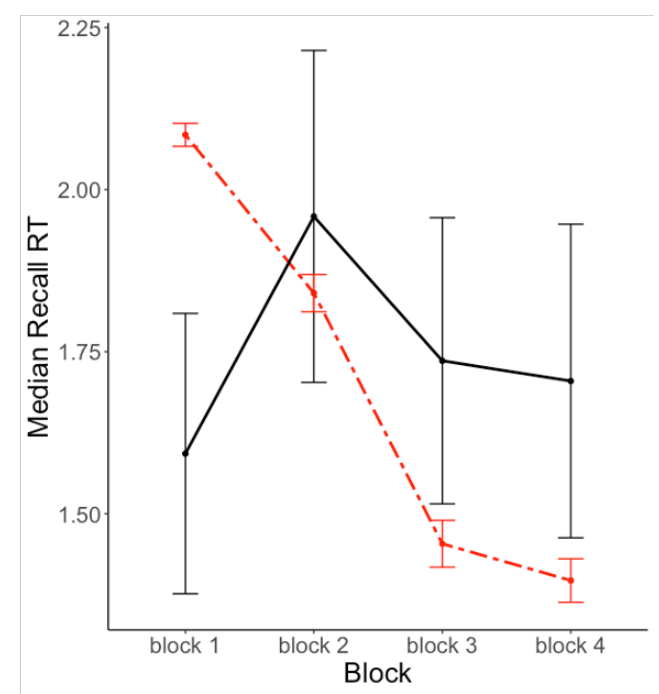

Figure S4: Block-wise recall performance in Experiment 1. Cued-recall performance broken down by block for a) accuracy and b) median RT.

\section{Experiment 2}

Results for this experiment were largely in line with the results from the previous experiment (Fig. S5). Participants recalled a higher proportion of names per block when they had been encoded with a meaningful English name tag compared to an Armenian tag $(F(1,38)=$ 17.70, $p<.001$, partial $\left.\eta^{2}=0.32\right)$. Participants also showed a change in performance over time $\left(F(3,114)=22.69, p<.001\right.$, partial $\left.\eta^{2}=0.37\right)$. Further investigation showed that participants scored lower on average recall during block 1 than on any other block $(p<.001$ for all comparisons with block 1), and the other blocks did not differ from one another $(p>.34$ for all comparisons). 
Reaction times for recall were significantly shorter for items initially presented with an English tag compared to those initially presented with an Armenian tag, $F(1,38)=7.42, p=$ .010. There was also a main effect of block $(F(3,114)=2.85, p=.05)$.

a)

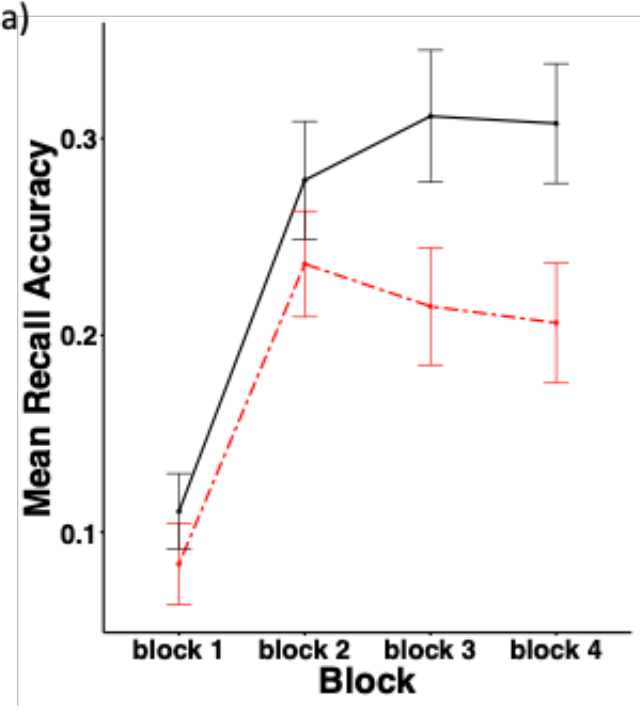

b)

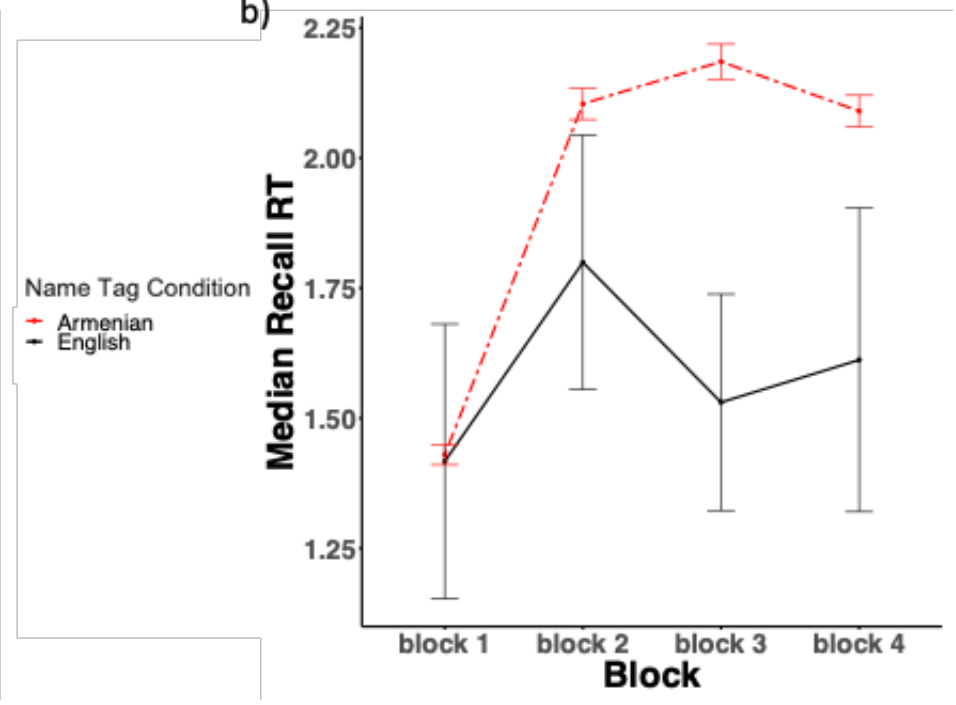

Figure S5: Block-wise recall performance in Experiment 2. Cued-recall performance broken down by block for a) accuracy and b) median RT (all responses).

\section{Experiment 3}

There was a significant main effect of the tag condition such that faces encoded with a name tag were associated with higher recall performance than those presented without a tag $(F(1,41)=21.53, p<.001$, partial $\eta 2=0.34$; Fig. S6a). There was also a main effect of block $(F(1,123)=21.15, p<.001$, partial $\eta 2=0.34)$, on which we ran post-hoc Tukey's HSD tests to investigate which blocks differed. Block 1 showed significantly lower performance than any other block ( $p$ <.001 for all comparisons with other blocks), but the other blocks did not differ significantly from one another ( $p>.27$ for all comparisons). The interaction of block and tag 
was non-significant $(F(3,123)=2.60, p=0.056)$. There was a statistically significant difference in median reaction times between the two tag conditions $(F(1,41)=14.22, p<.001)$, but no significant effect of block $(F(1,123)=0.72, p=.54$; Fig. S6b).

a)

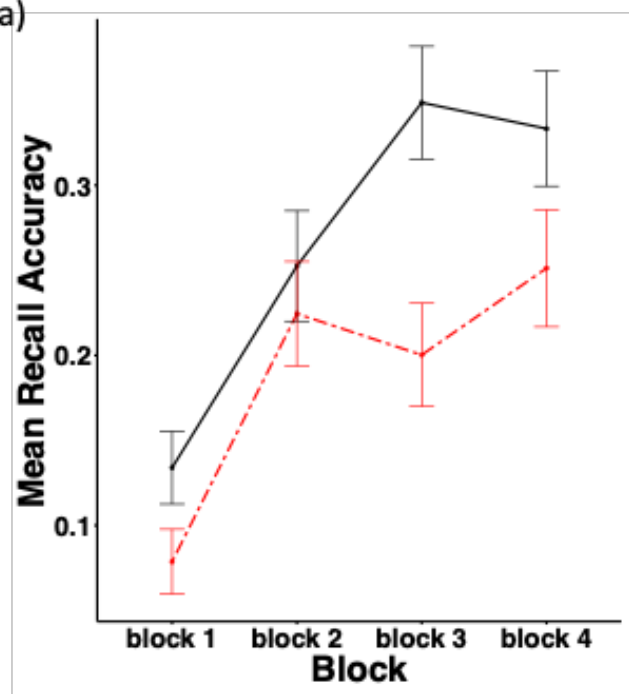

b)

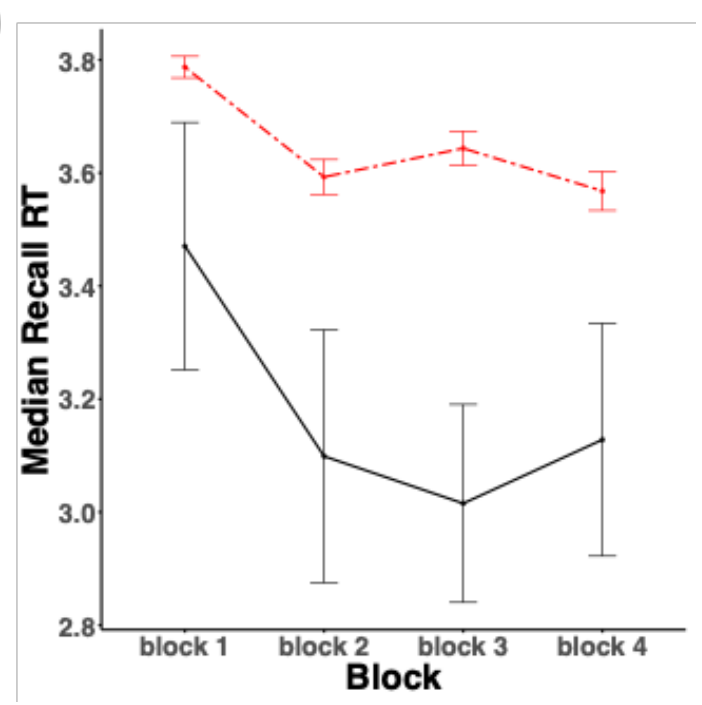

Figure S6: Block-wise recall performance in Experiment 3. Cued-recall performance broken down by block for a) accuracy and b) median RT (all responses).

\section{Experiment 4:}

Participants showed a higher average recall performance when presented with a name tag than when it was absent $(F(1,49)=9,66, p=.003$, partial $\eta 2=0.16)$ and showed a significant main effect of the block order $(F(5,245)=7.16, p<.001$, partial $\eta 2=0.13$; Fig. 7a). The interaction was nonsignificant $(F(5,245)=0.14, p=.98)$. Performance in block 1 was lower than in any other block ( $p=.008$ or less for every comparison), and other blocks did not differ significantly from one another ( $p=0.76$ or larger for every comparison). Thus, the effects observed in the previous experiments persisted even when the duration of the tag was better 


\section{MULTISENSORY ENCODING OF NAMES}

matched to the duration of the auditory signal. There was no statistically significant difference in reaction times between the two tag conditions $(F(1,49)=0.29, p=.59)$, though there was a significant effect of block $(F(1,245)=6.68, p<.001 ;$ Fig. $7 b)$. An analysis of this effect with a Tukey's HSD test showed that median RT in block 1 was higher than the median RT in block 3-6 ( $p \leq .006$ for all comparisons), with no other significant block differences ( $p \geq .13$ for all remaining comparisons).

Performance in the recognition task showed a similar trend (Fig. 7c). The main effect of block remained $(F(5,245)=7.59, p<.001$, partial $\eta 2=0.13)$, and there was a trend towards an effect of tag presentation $(F(1,49)=3.37, p=0.07)$, such that presentations with a tag were recognized better than those with no tag. Similar to recall, recognition performance was significantly lower in block 1 than in all other blocks ( $p<.001$ for all comparisons), and all other blocks did not differ significantly from one another ( $p=0.18$ or greater for all comparisons). There was no statistically significant difference in reaction times between the two tag conditions $(F(1,49)=2.66, p=.11 ; F i g .7 d)$, though there was a significant effect of block $(F(5$, $245)=3.28, p=.01)$. Post-hoc Tukey HSD tests showed that median RTs in block 1 were longer than those of blocks $4(p=.02)$ and block $6(p=.01)$, were marginally longer than RTs in blocks 3 , and $5(p=.10$ and $p=.10)$. No other block comparisons were significant $(p \geq .46$ for all comparisons). 

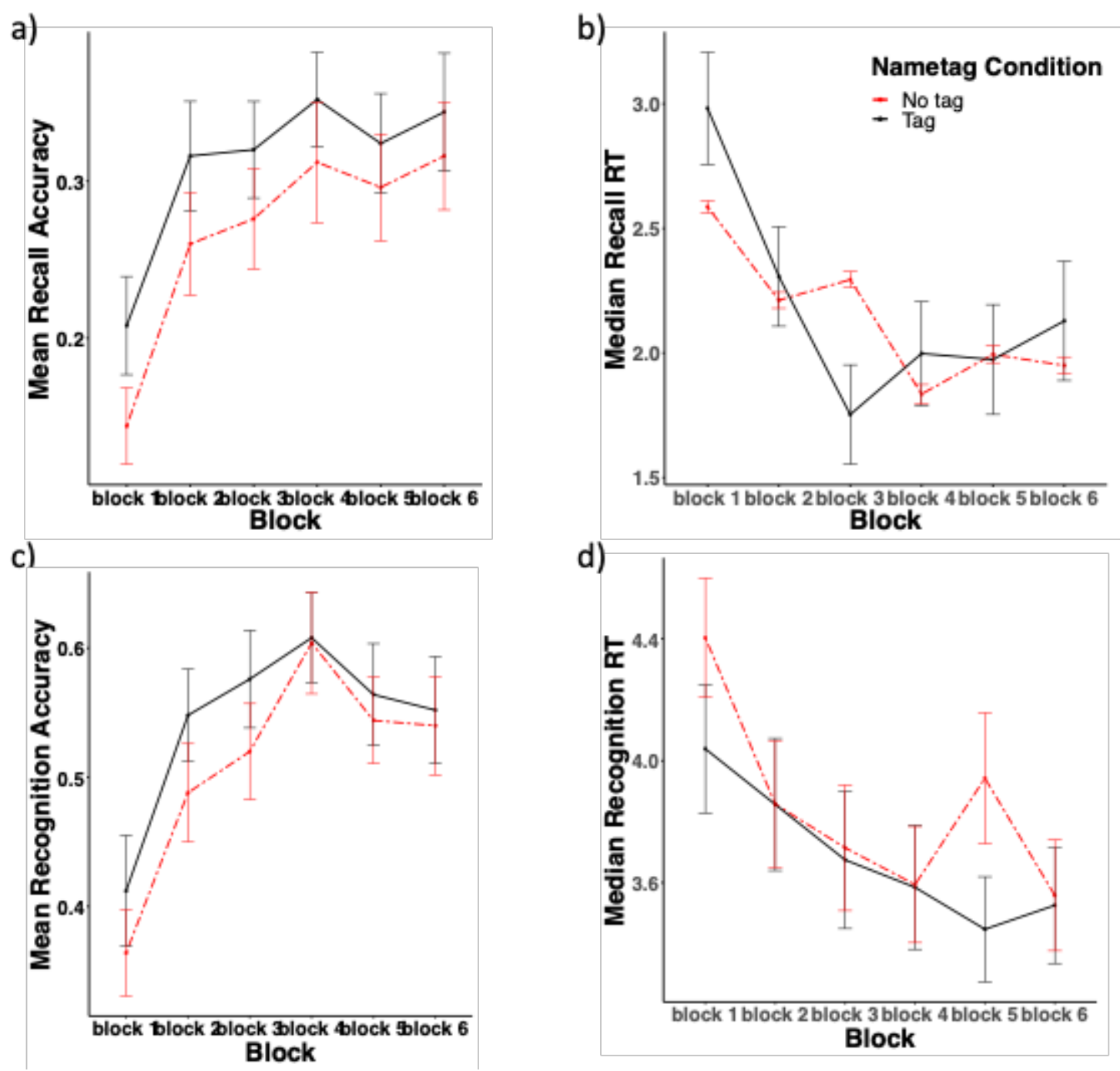

Figure S7: Block-wise recall performance in Experiment 4. Cued-recall performance broken down by block for a) accuracy and b) median RT (all responses), and recognition performance broken down by block for c) accuracy and d) median RT (all responses).

\section{Experiment 5:}

Participants showed a higher average recall performance when presented with a synchronous name tag than when it was asynchronous $(F(1,33)=5.14, p=.030$, partial $\eta 2=$ $0.13)$ and showed a significant main effect of the block order $(F(5,165)=7.01 p<.001$, partial 


\section{MULTISENSORY ENCODING OF NAMES}

$\eta 2=0.18$; Fig. S8a). Performance in block 1 was lower than in any other block except block 5 ( $p$ $=.039$ or below), and other blocks did not differ significantly from one another $(p=.057$ or above). Thus, the results support that multisensory representations or mechanisms, specifically, are supporting the improved performance, above any benefit from providing additional sensory traces as predicted by multiple memory traces.

There was no statistically significant difference in median recall reaction times between the two tag conditions $(F(1,33)=q .27, p=.27$; Fig. S8b), though there was a significant effect of block $(F(5,165)=5.38, p=.002 ;$ Fig. 8b). Post-hoc Tukey's HSD tests showed that median RT in block 1 was longer than that of blocks 2,3 , and 6 ( $p \leq .03$ for all comparisons), was marginally longer than the median RT of block $5(p=.08)$, and no other blocks significantly differed from one another ( $p \geq .18$ for all remaining comparisons).

Recognition results for this experiment showed no significant effect for tag condition $(F(1,33)=0.81, p=.37)$, but a significant effect of block $(F(5,165)=10.79, p<.001 ; F i g . S 8 c)$. Post-hoc Tukey's HSD tests showed that accuracy in block 1 was significantly lower than every other block ( $p \leq .001$ for all comparisons), while no other blocks differed significantly from one another ( $p \geq .51$ for all comparisons). Reaction time for recognition data showed a similar trend, such that tag condition was not significant $(F(1,33)=0.32, p=.58)$, while block was significant $(F(5,165)=5.33, p<.001 ;$ Fig. S8d). Post-hoc Tukey's HSD test showed that median recognition response times were longer in block 1 than in blocks $2-3$ and 6 ( $p \leq .03$ for all comparisons) and trended towards being longer than reaction times in block $4(p=.08)$. No other blocks differed significantly on median reaction times. 
a)

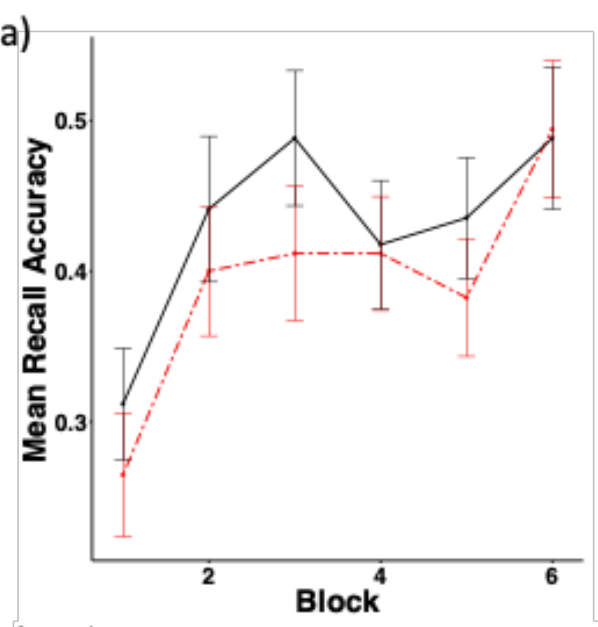

c)

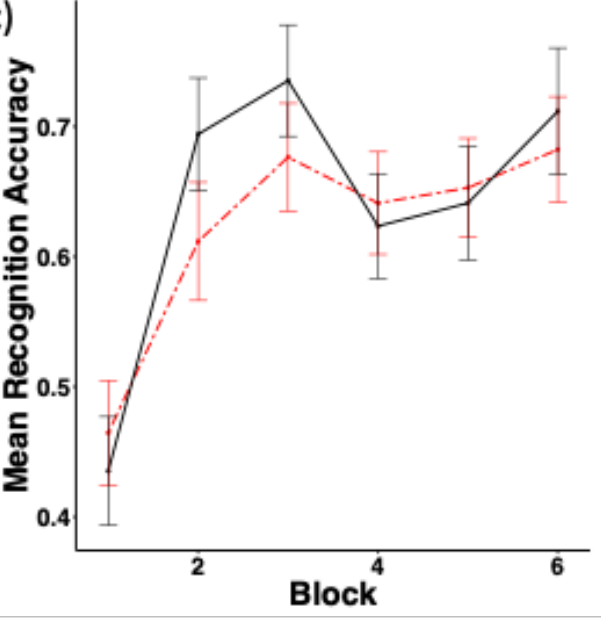

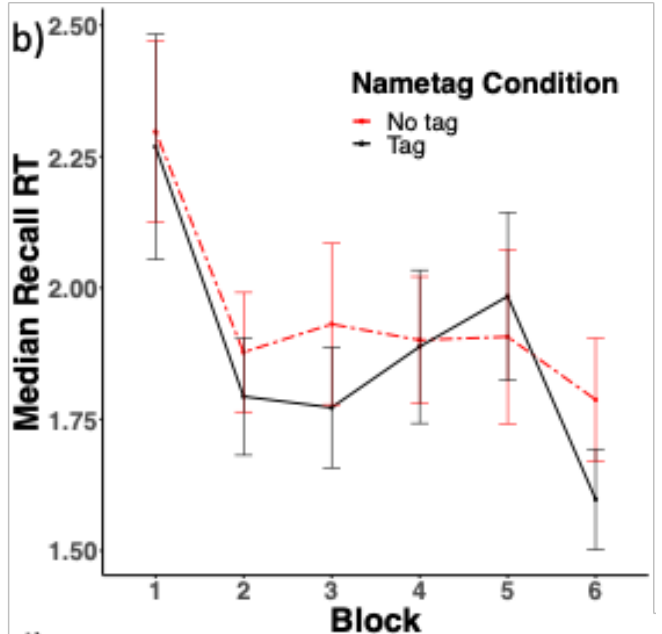

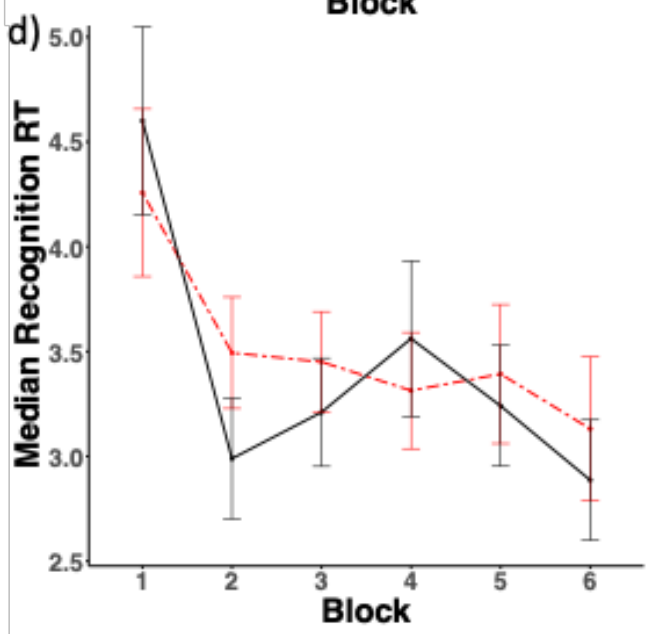

Figure S8: Block-wise recall performance in Experiment 5. Cued-recall performance broken down by block for a) accuracy and b) median $\mathrm{RT}$ (all responses), and recognition performance broken down by block for c) accuracy and d) median RT (all responses). 